\title{
CURVE COUNTING ON ELLIPTIC CALABI-YAU THREEFOLDS VIA DERIVED CATEGORIES
}

\author{
GEORG OBERDIECK AND JUNLIANG SHEN
}

\begin{abstract}
We prove the elliptic transformation law of Jacobi forms for the generating series of Pandharipande-Thomas invariants of an elliptic Calabi-Yau 3-fold over a reduced class in the base. This proves part of a conjecture by Huang, Katz, and Klemm. For the proof we construct an involution of the derived category and use wall-crossing methods. We express the generating series of PT invariants in terms of low genus Gromov-Witten invariants and universal Jacobi forms.

As applications we prove new formulas and recover several known formulas for the PT invariants of $\mathrm{K} 3 \times E$, abelian 3 -folds, and the STUmodel. We prove that the generating series of curve counting invariants for $\mathrm{K} 3 \times E$ with respect to a primitive class on the K3 is a quasi-Jacobi form of weight -10 . This provides strong evidence for the Igusa cusp form conjecture.
\end{abstract}

\section{Contents}

0. Introduction

1. Elliptic Calabi-Yau threefolds

2. $\pi$-stable pairs and wall-crossing

3. The derived equivalence $\Phi_{H}$

4. Applications

5. Examples

28

References

\section{INTRODUCTION}

0.1. Overview. Recently, considerations in topological string theory led Huang, Katz, and Klemm [16] to conjecture a deep connection between curve counting invariants of elliptic Calabi-Yau 3-folds and the theory of Jacobi forms [13]. Strong modular structure results for all genus are predicted.

On the other hand, derived categories of coherent sheaves play a crucial role in counting curves by the work of Pandharipande and Thomas [32].

Date: November 14, 2019. 
Symmetries in derived categories are expected to affect curve counting invariants. More precisely, Toda asks in [37] the following question.

Question 1. How are curve counting invariants on a Calabi-Yau 3-fold constrained due to the presence of non-trivial derived auto-equivalences?

The purpose of this paper is to study Question 1 for elliptic Calabi-Yau 3 -folds and explore its connection to the modular constraints of the HuangKatz-Klemm conjecture. We prove that a specific auto-equivalence induces the elliptic transformation law of Jacobi forms on generating series. By further combining techniques from Jacobi forms, Pandharipande-Thomas theory, and Gromov-Witten theory this yields new practical computation methods for curve counting invariants. In particular we obtain strong evidence for the Igusa cusp form conjecture [27].

0.2. Elliptic fibrations. Let $X$ be a non-singular projective threefold satisfying

$$
\omega_{X} \simeq \mathcal{O}_{X} \quad \text { and } \quad H^{1}\left(X, \mathcal{O}_{X}\right)=0 .
$$

Let $S$ be a non-singular projective surface and assume $X$ admits an elliptic fibration over $S$ - a flat and proper morphism

$$
\pi: X \rightarrow S
$$

with fibers reduced and irreducible curves of arithmetic genus 1. The fibers of $\pi$ are therefore one of the following types:

(i) a non-singular elliptic curve,

(ii) a nodal rational curve (a cubic in $\mathbb{P}^{2}$ with a nodal singularity),

(iii) a cuspidal rational curve (a cubic in $\mathbb{P}^{2}$ with a cuspidal singularity).

We further assume the fibration $\pi$ has a section

$$
\iota: S \rightarrow X, \quad \pi \circ \iota=\mathrm{id}_{S} .
$$

0.3. Stable pairs. A stable pair $(\mathcal{F}, s)$ on $X$ is a coherent sheaf $\mathcal{F}$ supported in dimension 1 and a section $s \in H^{0}(X, \mathcal{F})$ satisfying the following stability conditions:

(i) the sheaf $\mathcal{F}$ is pure

(ii) the cokernel of $s$ is 0 -dimensional.

To a stable pair we associate the Euler characteristic and the class of the support $C$ of $\mathcal{F}$,

$$
\chi(\mathcal{F})=n \in \mathbb{Z} \quad \text { and } \quad[C]=\beta \in H_{2}(X, \mathbb{Z}) .
$$

Let $P_{n}(X, \beta)$ be the moduli space of stable pairs of given numerical type. Pandharipande-Thomas invariants 32 are defined by integrating the Behrend 
function 3

$$
\nu: P_{n}(X, \beta) \rightarrow \mathbb{Z}
$$

with respect to the topological Euler characteristic $e(\cdot)$ over the moduli space:

$$
\mathrm{P}_{n, \beta}=\int_{P_{n}(X, \beta)} \nu \mathrm{d} e=\sum_{k \in \mathbb{Z}} k \cdot e\left(\nu^{-1}(k)\right) .
$$

The set of invariants $P_{n, \beta}$ are intricately related to the number and type of algebraic curves in $X$ [33].

0.4. Elliptic transformation law. Let $H \in \operatorname{Pic}(S)$ be an effective divisor class of arithmetic genus

$$
h=1+\frac{1}{2}\left(H^{2}+K_{S} \cdot H\right) .
$$

Let $F \in H_{2}(X, \mathbb{Z})$ be the class of a fiber of $\pi$, and consider the curve classes

$$
H+d F:=\iota_{*}(H)+d F \in H_{2}(X, \mathbb{Z}), \quad d \geq 0,
$$

where we have suppressed the cycle class map that takes the divisor $H$ to its class in homology. We define the generating series of stable pairs invariants,

$$
\mathrm{PT}_{H}(q, t)=\sum_{d=0}^{\infty} \sum_{n \in \mathbb{Z}} \mathrm{P}_{n, H+d F} q^{n} t^{d} .
$$

By a calculation of Toda [36, Thm 6.9] we have a complete evaluation in case $H=0$,

$$
\mathrm{PT}_{0}(q, t)=\prod_{\ell, m \geq 1}\left(1-(-q)^{\ell} t^{m}\right)^{-\ell \cdot e(X)} \cdot \prod_{m \geq 1}\left(1-t^{m}\right)^{-e(S)} .
$$

We consider here the case when $H$ is reduced, that is, if in every decomposition $H=\sum_{i} H_{i}$ into effective classes, all of the $H_{i}$ are primitive.

The following is the main result of the paper.

Theorem 1. Let $H \in \operatorname{Pic}(S)$ be reduced of arithmetic genus $h$. Then the following equality of generating series holds:

$$
\frac{\mathrm{PT}_{H}\left(q^{-1} t, t\right)}{\mathrm{PT}_{0}\left(q^{-1} t, t\right)}=q^{2(h-1)} t^{-(h-1)} \frac{\mathrm{PT}_{H}(q, t)}{\mathrm{PT}_{0}(q, t)} .
$$

Let $\mathrm{Z}_{H}(q, t)=\mathrm{PT}_{H}(q, t) / \mathrm{PT}_{0}(q, t)$. Then Theorem 1 can be rewritten as

$$
\mathrm{Z}_{H}\left(q^{-1} t, t\right)=q^{2(h-1)} t^{-(h-1)} \mathrm{Z}_{H}(q, t) .
$$

By [5, 38] every series $\sum_{n \in \mathbb{Z}} \mathrm{P}_{n, H+d F} q^{n}$ is the Laurent expansion of a rational function in $q$ invariant under the variable change $q \mapsto q^{-1}$. Considering $\mathrm{Z}_{H}(q, t)$ as an element in $\mathbb{Q}(q)[[t]]$, we therefore also have

$$
\mathrm{Z}_{H}\left(q^{-1}, t\right)=\mathrm{Z}_{H}(q, t) \text {. }
$$


Combining with Theorem 1 we obtain the following relationship of $Z_{H}$ to the theory of Jacobi forms [13].

Corollary 1. Let $H \in \operatorname{Pic}(S)$ be reduced of arithmetic genus $h$. Then $\mathrm{Z}_{H}(q, t) \in \mathbb{Q}(q)[[t]]$ satisfies the elliptic transformation law for Jacobi forms of index $h-1$, that is, for all $\lambda \in \mathbb{Z}$

$$
\mathrm{Z}_{H}\left(q t^{\lambda}, t\right)=t^{-(h-1) \lambda^{2}} q^{-2(h-1) \lambda} \mathbf{Z}_{H}(q, t) .
$$

To emphasize, the equality of Corollary 1 holds only as an identity of elements in $\mathbb{Q}(q)[[t]]$. In contrast, Theorem 1 is an equality of generating series and yields an identity on the level of coefficients.

By physical considerations and explicit calculations, Huang, Katz and Klemm conjecture the series $Z_{H}(q, t)$ to be a meromorphic Jacobi form of index $h-1$ [16]. Jacobi forms must satisfy two different equations: the elliptic and the modular transformation law. Corollary 1 therefore proves exactly half of the conjecture of Huang-Katz-Klemm in case $H$ is reduced 1 . We will come back to this below.

0.5. Genus zero. Let $\mathcal{M}_{\beta}$ be the moduli space of one-dimensional stable sheaves $\mathcal{F}$ with $\chi(\mathcal{F})=1$ and $\operatorname{ch}_{2}(\mathcal{F})=\beta$. Following [17] the genus 0 Gopakumar-Vafa invariant in class $\beta$ is the Behrend function weighted Euler characteristic

$$
\mathrm{n}_{\beta}=\int_{\mathcal{M}_{\beta}} \nu \mathrm{d} e .
$$

The invariant $\mathbf{n}_{\beta}$ is a virtual count of rational curves in class $\beta$.

We define the genus 0 potential in classes $H+d F$ by

$$
\mathrm{F}_{H}(t)=\sum_{d \geq 0} \mathrm{n}_{H+d F} t^{d}
$$

Let also

$$
\phi_{-2,1}(q, t)=\left(q+2+q^{-1}\right) \prod_{m \geq 1} \frac{\left(1+q t^{m}\right)^{2}\left(1+q^{-1} t^{m}\right)^{2}}{\left(1-t^{m}\right)^{4}}
$$

be the (up to scaling) unique weak Jacobi form of weight -2 and index 1 , see [13, Thm 9.3]2.

Theorem 2. Let $H \in \operatorname{Pic}(S)$ be irreducible of arithmetic genus $h=0$. Then

$$
\frac{\mathrm{PT}_{H}(q, t)}{\mathrm{PT}_{0}(q, t)}=\mathrm{F}_{H}(t) \cdot \frac{1}{\phi_{-2,1}(q, t)}
$$

\footnotetext{
1 The non-reduced case of Theorem 1 will be considered in 28 .

2 The variables $z \in \mathbb{C}, \tau \in \mathbb{H}$ of $\left[13\right.$ are related to $(q, t)$ by $q=e^{2 \pi i(z+1 / 2)}$ and $t=e^{2 \pi i \tau}$. Also our convention of $\phi_{-2,1}$ (but not $\phi_{0,1}$ below) differs from [13] by a sign.
} 
If $H$ is irreducible of genus 0 then every curve in $X$ of class $H+d F$ consists of a section over a line in the base together with vertical components. Theorem 2 then says that the series $\mathrm{PT}_{H}$ is a genus 0 term (counting sections) times a universal contribution coming from the fiber geometry.

0.6. General case. We have the following more general structure result.

Consider the Weierstraß elliptic function

$$
\wp(q, t)=-\frac{1}{12}+\frac{q}{(1+q)^{2}}-\sum_{d \geq 1} \sum_{m \mid d} m\left((-q)^{m}-2+(-q)^{-m}\right) t^{d}
$$

and let

$$
\phi_{0,1}(q, t)=12 \wp(q, t) \cdot \phi_{-2,1}(q, t)
$$

be the unique weak Jacobi form of weight 0 and index 1 .

Theorem 3. Let $H \in \operatorname{Pic}(S)$ be a reduced class of arithmetic genus $h \geq 0$, and let $n$ be the largest integer for which there exist a decomposition $H=$ $\sum_{i=1}^{n} H_{i}$ into effective classes. Then there exist power series

$$
f_{-(n-1)}(t), \ldots, f_{h}(t) \in \mathbb{Q}[[t]]
$$

such that

$$
\frac{\mathrm{PT}_{H}(q, t)}{\mathrm{PT}_{0}(q, t)}=\sum_{i=-(n-1)}^{h} f_{i}(t) \cdot \phi_{-2,1}(q, t)^{i-1} \phi_{0,1}(q, t)^{h-i} .
$$

By the conjecture of Huang, Katz and Klemm [16], we expect $\mathrm{PT}_{H} / \mathrm{PT}_{0}$ to be a meromorphic Jacobi form of index $h-1$ and some weight $\ell$. This is equivalent to saying that every

$$
f_{i}(t) \in \mathbb{Q}[[t]]
$$

in Theorem 3 is a weak modular form of weight $\ell+2 i-2$.

Similar to before we may think of the functions $f_{i}(t)$ as counting generalized genus $i$ sections over curves in the base 3 . Concretely, by [16] and the examples of Section 5 for every $i$ there should be a natural splitting

$$
f_{i}(t)=\prod_{m \geq 1} \frac{1}{\left(1-t^{m}\right)^{-12\left(K_{S} \cdot H\right)}} \cdot g_{i}(t) .
$$

By calculations of Bryan and Leung [9] the factor

$$
\prod_{m \geq 1} \frac{1}{\left(1-t^{m}\right)^{-12\left(K_{S} \cdot H\right)}}
$$

is the contribution to $f_{i}(t)$ of a fixed curve with no vertical components. The remaining factor

$$
g_{i}(t) \in \mathbb{Q}[[t]]
$$

\footnotetext{
3 The genus can be negative here if the curve is disconnected.
} 
is expected to be a modular form related to the jumping of the Picard rank in the fibers of the family

$$
\pi^{-1}(L), \quad L \in|H|
$$

We hope that an approach using Noether-Lefschetz theory [22] can provide a pathway to the modularity of $g_{i}(t)$.

0.7. Gromov-Witten theory. We now assume that $X$ satisfies the GW/PT correspondence which relates Pandharipande-Thomas invariants to GromovWitten invariants of $X$, see [32, Conj 3.3]. By [30, 31] the correspondence holds when $X$ is a complete intersection in a product of projective spaces.

The genus $g$ Gromov-Witten invariant of $X$ is defined by the integral

$$
\mathrm{GW}_{g, \beta}=\int_{\left[\bar{M}_{g}(X, \beta)\right]^{\mathrm{vir}}} 1
$$

where $\bar{M}_{g}(X, \beta)$ is the moduli space of genus $g$ stable maps to $X$ with connected domain, and $[\cdot]^{\text {vir }}$ is its virtual class. Define the generating series of genus $g$ Gromov-Witten invariants

$$
\mathrm{GW}_{H}^{g}(t)=\sum_{d \geq 0} \mathrm{GW}_{g, H+d F} t^{d} .
$$

Proposition 1. Assume the GW/PT correspondence holds for $X$, and let $H \in \operatorname{Pic}(S)$ be irreducible of arithmetic genus $h$.

Then the series $f_{i}(t)$ of Theorem 3 are effectively determined from the series $\mathrm{GW}_{H}^{i}(t), i=0, \ldots, h$ via the equality

(3) $\sum_{i=0}^{h} f_{i}(t) \phi_{-2,1}(q, t)^{i-1} \phi_{0,1}(q, t)^{h-i} \equiv \sum_{g=0}^{h} \mathrm{GW}_{H}^{g}(t) u^{2 g-2} \bmod u^{2 h}$

under the variable change $q=-e^{i u}$.

By inverting the system (3) we find $\mathrm{PT}_{H}$ is an universal linear combination of the first $h+1$ Gromov-Witten series. In particular the GromovWitten invariants up to genus $h$ determine the Gromov-Witten invariants of arbitrary genus. As examples we consider the first few cases.

Under the assumptions of Theorem 3 the genus 0 Gopakumar-Vafa invariants agree with the genus 0 Gromov-Witten invariants:

$$
\mathrm{n}_{H+d F}=\mathrm{GW}_{0, H+d F} .
$$

Hence in genus $h=0$ we recover Theorem 2, In genus $h=1$ Theorem 3 yields

$$
\frac{\mathrm{PT}_{H}(q, t)}{\mathrm{PT}_{0}(q, t)}=\mathrm{GW}_{H}^{0}(t) \cdot \wp(q, t)+\mathrm{GW}_{H}^{1}(t)
$$


To state the genus 2 case, let

$$
E_{2 k}(t)=1-\frac{4 k}{B_{2 k}} \sum_{d \geq 1} \sum_{m \mid d} m^{2 k-1} t^{d}
$$

be the Eisenstein series with $B_{2 k}$ the Bernoulli numbers. Then, for $h=2$,

$$
\begin{aligned}
\frac{\mathrm{PT}_{H}}{\mathrm{PT}_{0}}= & \mathrm{GW}_{H}^{0} \cdot\left(\wp^{2} \phi_{-2,1}+\frac{1}{12} E_{2} \wp \phi_{-2,1}+\left(\frac{1}{288} E_{2}^{2}-\frac{11}{1440} E_{4}\right) \phi_{-2,1}\right) \\
& +\mathrm{GW}_{H}^{1} \cdot\left(\wp \phi_{-2,1}+\frac{1}{12} E_{2} \phi_{-2,1}\right) \\
& +\mathrm{GW}_{H}^{2} \cdot \phi_{-2,1} .
\end{aligned}
$$

where we omitted the dependence on $q, t$.

0.8. An example: $\mathrm{K} 3 \times E$. Let $S$ be a non-singular projective $\mathrm{K} 3$ surface, and let $E$ be an elliptic curve. Consider the product Calabi-Yau $X=S \times E$ elliptically fibered along the projection to the first factor,

$$
\pi: X \longrightarrow S \text {. }
$$

Let $0_{E} \in E$ be the zero and fix the section

$$
\iota: S \rightarrow X, s \mapsto\left(s, 0_{E}\right) .
$$

For a non-zero class $H \in \operatorname{Pic}(S)$ the group $E$ acts on the moduli space $P_{n}(X, H+d F)$ by translation with finite stabilizers. Reduced PandharipandeThomas invariants of $X$ are defined by integrating the Behrend function $\nu$ over the quotient space

$$
\mathrm{P}_{n, H+d F}^{\mathrm{red}}=\int_{P_{n}(X, H+d F) / E} \nu \mathrm{d} e
$$

where the Euler characteristic is taken in the orbifold sense. We define the generating series of reduced invariants

$$
\mathrm{PT}_{H}^{\mathrm{red}}(q, t)=\sum_{d \geq 0} \sum_{n \in \mathbb{Z}} \mathrm{P}_{n, H+d F}^{\mathrm{red}} q^{n} t^{d} .
$$

If $H$ is primitive, the series $\mathrm{PT}_{H}^{\mathrm{red}}(q, t)$ depends by deformation invariance only on the arithmetic genus $h$ of $H$ and we write

$$
\mathrm{PT}_{h}^{\mathrm{K} 3 \times E}(q, t)=\mathrm{PT}_{H}^{\mathrm{red}}(q, t) .
$$

The ring QMod of holomorphic quasi-modular forms is the free polynomial algebra in the Eisenstein series $E_{2}(t), E_{4}(t)$ and $E_{6}(t)$,

$$
\mathrm{QMod}=\mathbb{Q}\left[E_{2}, E_{4}, E_{6}\right] .
$$

Recall the Jacobi forms $\phi_{-2,1}(q, t)$ and $\phi_{0,1}(q, t)$. The ring

$$
\widetilde{\operatorname{Jac}}=\mathrm{QMod}\left[\phi_{-2,1}, \phi_{0,1}\right]
$$


carries a natural bigrading by index and weight,

$$
\widetilde{\mathrm{Jac}}=\bigoplus_{m \geq 0} \bigoplus_{k \geq-2 m}{\widetilde{\mathrm{Jac}_{k, m}}}_{\text {, }}
$$

where $E_{2 k}$ has weight $2 k$ and index 0 , and $\phi_{k, 1}$ has weight $k$ and index 1 .

Define the modular descriminant

$$
\Delta(t)=\prod_{m \geq 1}\left(1-t^{m}\right)^{24}
$$

Theorem 4. We have

$$
\mathrm{PT}_{h}^{\mathrm{K} 3 \times E}(q, t)=\frac{\Psi_{h}(q, t)}{\Delta(t) \phi_{-2,1}(q, t)}
$$

for a series $\Psi_{h}(q, t) \in \widetilde{\mathrm{Jac}}_{0, h}$ of index $h$ and weight 0 .

Hence in the language of [24] we find $\mathrm{PT}_{h}^{\mathrm{K} 3 \times E}(q, t)$ is a quasi-Jacobi form of index $h-1$ and weight -10 . In particular the full series $\mathrm{PT}_{h}^{\mathrm{K} 3 \times E}$ is determined from finitely many coefficients.

By basic Gromov-Witten calculations we recover a result of Bryan [7].

\section{Theorem 5.}

$$
\begin{aligned}
\mathrm{PT}_{0}^{\mathrm{K} 3 \times E}(q, t) & =\frac{1}{\Delta(t) \phi_{-2,1}(q, t)} \\
\mathrm{PT}_{1}^{\mathrm{K} 3 \times E}(q, t) & =\frac{24 \wp(q, t)}{\Delta(t)}
\end{aligned}
$$

A complete evaluation of the invariants $\mathrm{P}_{n, H+d F}^{\mathrm{red}}$ was conjectured in [27], motivated by physical predictions [18] and the calculations [24]. For primitive $H$ the conjecture takes the form

$$
\sum_{h=0}^{\infty} \mathrm{PT}_{h}^{\mathrm{K} 3 \times E}(q, t) u^{h}=\frac{1}{\chi_{10}(q, t, u)}
$$

where $\chi_{10}$ is the Igusa cusp form - a Siegel modular form of weight 10 . Theorem 5 verifies this conjecture in cases $h=0$ and $h=1$, and Theorem 4 gives strong evidence for every genus $h$.

0.9. Euler characteristics. The proof of Theorem 1 is based on wallcrossing techniques and applies also for the (unweighted) Euler characteristic of the moduli spaces. We state parallel results for the unweighted case.

Define naïve Pandharipande-Thomas invariants as the Euler characteristic of the moduli space of stable pairs,

$$
\widetilde{\mathrm{P}}_{n, \beta}=e\left(P_{n}(X, \beta)\right) \text {. }
$$

\footnotetext{
${ }^{4}$ We modify here the usual definition of $\Delta$ by a shift of $t$ to avoid making a similar shift in the definition of $\mathrm{PT}_{H}$.
} 
We form the generating series

$$
\widetilde{\mathrm{PT}}_{H}(p, t)=\sum_{d=0}^{\infty} \sum_{n \in \mathbb{Z}} \widetilde{\mathrm{P}}_{n, H+d F} p^{n} t^{d}
$$

where we use the variable $p$ instead of $q$. By the same argument as in Toda's calculation [36] we have

$$
\widetilde{\mathrm{PT}}_{0}(p, t)=\prod_{\ell, m \geq 1}\left(1-p^{\ell} t^{m}\right)^{-\ell \cdot e(X)} \cdot \prod_{m \geq 1}\left(1-t^{m}\right)^{-e(S)} .
$$

Theorem 6. Let $H \in \operatorname{Pic}(S)$ be reduced of arithmetic genus $h$. Then we have the equality of generating series

$$
\frac{\widetilde{\mathrm{PT}}_{H}\left(p^{-1} t, t\right)}{\widetilde{\mathrm{PT}}_{0}\left(p^{-1} t, t\right)}=p^{2(h-1)} t^{-(h-1)} \frac{\widetilde{\mathrm{PT}}_{H}(p, t)}{\widetilde{\mathrm{PT}}_{0}(p, t)} .
$$

Moreover, let $n$ be the largest integer for which there exist an effective decomposition $H=\sum_{i=1}^{n} H_{i}$. Then there exist $\tilde{f}_{i}(t) \in \mathbb{Q}[[t]]$ such that

$$
\frac{\widetilde{\mathrm{PT}}_{H}(p, t)}{\widetilde{\mathrm{PT}}_{0}(p, t)}=\sum_{i=-(n-1)}^{h} \tilde{f}_{i}(t) \cdot \phi_{-2,1}(-p, t)^{i-1} \phi_{0,1}(-p, t)^{h-i} \text {. }
$$

In case of (honest) $\mathrm{PT}$ invariants we expected $\mathrm{PT}_{H}(q, t)$ to be a Jacobi form and the functions $f_{k}(t)$ to be modular forms. As we will see in Section 5 in the example of abelian threefolds, the same does not hold for $\widetilde{f}_{k}(t)$.

Theorem 6 implies that the naïve and the honest Pandharipande-Thomas invariants of $X$ are closely related under the variable change $q=-p$. The only difference arises from counting the section terms differently and the Behrend function does not seem to play any larger role. Our results are therefore in non-trivial agreement with previous observations and conjectures on the Behrend function in elliptic geometries [7, 8, 10].

0.10. Idea of the proof of Theorem 1, The identity of Theorem 1 arises from two separate steps: applying a derived equivalence $\Phi_{H}$ to the moduli space of stable pairs, followed by a wall-crossing in the motivic Hall algebra.

The derived equivalence $\Phi_{H}$ sends a stable pair $\mathcal{O}_{X} \rightarrow \mathcal{F}$ to a $\pi$-stable pair, which is a modification of the usual definition of stable pairs adapted to the elliptic geometry. If the stable pair has numerical invariants $(H+d F, n)$ then the transformed $\pi$-stable pair has invariants

$$
(H+\widetilde{d} F, \widetilde{n})=(H+(h+d+n-1) F,-n-2 h+2) .
$$

The definition of $\pi$-stable pairs is similar in philosophy to the modification of stable pairs by Bryan-Steinberg [11] for a crepant resolution $X \rightarrow Y$ that contracts an exceptional curve. While for BS-pairs we allow a pair $\mathcal{O}_{X} \rightarrow \mathcal{F}$ to have 1-dimensional cokernel along the exceptional curve, here we allow a 
$\pi$-stable pair to have 1-dimensional cokernel supported on arbitrary fibers of the fibration. A wall-crossing argument following Toda 38 relates $\pi$ stable pairs invariants to usual Pandharipande-Thomas invariants with a correction term involving the count of semistable sheaves supported on fibers of $\pi$. This yields naturally the term $\mathrm{PT}_{0}$ in the equation of Theorem 1 .

The derived auto-equivalence $\Phi_{H}$ also arises naturally from the elliptic fibration $\pi$. Consider the fibered product

$$
X \times{ }_{S} X
$$

over the base $S$, and let $\mathcal{I}_{\Delta}^{*}$ be the dual of the ideal sheaf of the diagonal in $X \times_{S} X$. Up to a normalization $\mathcal{I}_{\Delta}^{*}$ is the Poincaré sheaf of the elliptic fibration. Let also

$$
X \stackrel{q}{\leftarrow} X \times_{S} X \stackrel{p}{\rightarrow} X
$$

be the natural projections to the first and second factor. The Fourier-Mukai transform $\phi_{\mathcal{I}_{\Delta}^{*}}$ with kernel $\mathcal{I}_{\Delta}^{*}$ is

$$
\phi_{\mathcal{I}_{\Delta}^{*}}(\mathcal{E})=R q_{*}\left(p^{*}(\mathcal{E}) \otimes \mathcal{I}_{\Delta}^{*}\right), \quad \mathcal{E} \in D^{b} \operatorname{Coh}(X) .
$$

For a line bundle $\mathcal{L} \in \operatorname{Pic}(X)$ let

$$
\mathbb{T}_{\mathcal{L}}(\mathcal{E})=\mathcal{L} \otimes \mathcal{E}
$$

be the twist by $\mathcal{L}$ and let $\mathbb{D}: D^{b} \operatorname{Coh}(X) \rightarrow D^{b} \operatorname{Coh}(X)$ be the dual functor,

$$
\mathbb{D}(\mathcal{E})=R \mathscr{H}^{\circ} m_{X}\left(\mathcal{E}, \mathcal{O}_{X}\right) .
$$

The auto-equivalence $\Phi_{H}$ is then defined as the composition

$$
\Phi_{H}=\mathbb{D} \circ \mathbb{T}_{\pi^{*} \mathcal{O}_{S}(H)} \circ \phi_{\mathcal{I}_{\Delta}^{*}} .
$$

The strategy of the proof is summarized in the following diagram, where $P_{n}^{\pi}(X, \beta)$ denotes the moduli space of $\pi$-stable pairs.

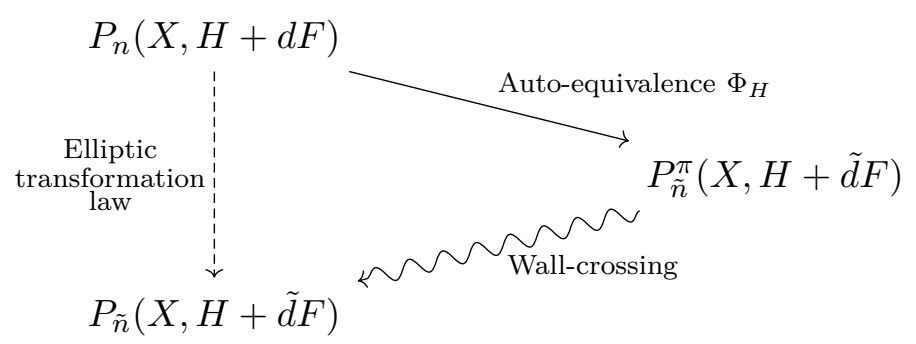

0.11. Plan of the paper. In Section 1 we recall several basic facts on elliptic fibrations and study sheaves supported on fibers of $\pi$. In Section 2 we introduce $\pi$-stable pairs and prove the wall-crossing. In Section 3 we study the derived equivalence. In particular, if $H$ is reduced, we prove a complex $I^{\bullet}$ is a stable pair if and only if $\Phi_{H}\left(I^{\bullet}\right)$ is a $\pi$-stable pair. This completes the proof of Theorem 1 In Section 4 we prove Theorems 2 and 3 , 
and the corresponding Euler characteristic case. In Section 5 we apply our methods to several examples including $\mathrm{K} 3 \times E$ and abelian threefolds.

0.12. Conventions. We always work over $\mathbb{C}$. For a variety $X$, the canonical bundle (or sheaf) is denoted $\omega_{X}$, and the canonical divisor is $K_{X}=c_{1}\left(\omega_{X}\right)$. The skyscraper sheaf at a point $x \in X$ is $\mathbb{C}_{x}$. The dual of a sheaf $\mathcal{F}$ is $\mathcal{F}^{*}=\mathscr{H} m_{X}\left(\mathcal{F}, \mathcal{O}_{X}\right)$, and the derived dual of a complex $\mathcal{E} \in D^{b} \operatorname{Coh}(X)$ is $\mathcal{E}^{\vee}=R \mathscr{H} m_{X}\left(\mathcal{E}, \mathcal{O}_{X}\right)$. If $i: X \hookrightarrow Y$ is a closed embedding and $\mathcal{F}$ is a sheaf on $X$, then we write $\mathcal{F}$ also for the pushforward $i_{*} \mathcal{F}$ on $Y$. For a sheaf $\mathcal{F}$ and a divisor $D$ on $X$, we let $\mathcal{F}(D)=\mathcal{F} \otimes \mathcal{O}_{X}(D)$.

0.13. Acknowledgements. The paper was started when both authors were attending the workshop Curves on surfaces and threefolds at the Bernoulli center at EPFL Lausanne in June 2016. Discussions with J. Bryan on elliptic geometries and the paper [11] were extremely helpful. We would also like to thank F. Greer, S. Katz, M. Kool, O. Leigh, D. Maulik, T. Padurariu, R. Pandharipande, Y. Toda, and Q. Yin for useful discussions.

J. S. was supported by grant ERC-2012-AdG-320368-MCSK in the group of R. Pandharipande at ETH Zürich.

\section{Elliptic Calabi-Yau threefolds}

1.1. Definition. Let $X$ be a Calabi-Yau 3-fold - a non-singular projective threefold with trivial canonical bundle $\omega_{X} \simeq \mathcal{O}_{X}$ and $H^{1}\left(X, \mathcal{O}_{X}\right)=0$. Let $S$ be a non-singular projective surface and let

$$
\pi: X \rightarrow S
$$

be an elliptic fibration with reduced and irreducible fibers. Hence fibers

$$
X_{s}=\pi^{-1}(s), s \in S
$$

are either non-singular elliptic curves or rational curves with a single node or cusp. Let further

$$
\iota: S \rightarrow X, \quad \pi \circ \iota=\operatorname{id}_{S}
$$

be a section of $\pi$. Necessarily, the section meets every fiber $X_{s}$ in a nonsingular point. We let

$$
0_{s}=\iota(s) \in X_{s}
$$

denote the distinguished point of the fiber $X_{s}$ over $s \in S$ and

$$
S_{0}:=\iota_{*} S
$$

the divisor on $X$ defined by the section. 
1.2. Compactified Jacobian. Let $\hat{X}$ be the relative compactified Jacobian of $X$ parametrizing torsion free, rank 1 and degree 0 sheaves on a fiber of $\pi: X \rightarrow S$. Since $\hat{X}$ is a fine moduli space, there exists a universal Poincaré sheaf on

$$
X \times_{S} \hat{X}
$$

uniquely defined up to tensoring by a line bundle pulled back from $\hat{X}$. We let $\mathcal{P}$ be the unique Poincaré sheaf satisfying the normalization

$$
\left.\mathcal{P}\right|_{S_{0} \times{ }_{S} \hat{X}} \simeq \mathcal{O}_{\hat{X}}
$$

The sheaves $\mathcal{P}$ and $\mathcal{P}^{*}$ are flat over both $X$ and $\hat{X}$, and $\mathcal{P}^{\vee}=\mathcal{P}^{*}[6,8.4]$.

Since $\pi$ admits a section and has integral fibers, we will identify $X$ with its compactified Jacobian $\hat{X}$ via the natural isomorphism

$$
X \stackrel{\cong}{\longrightarrow}, \quad x \mapsto \imath_{s *}\left(\mathfrak{m}_{x}^{*} \otimes \mathcal{O}_{X_{s}}\left(-0_{s}\right)\right)
$$

where $\imath_{s}: X_{s} \hookrightarrow X$ is the inclusion of the fiber over $s=\pi(x)$ and $\mathfrak{m}_{x}$ is the ideal sheaf of $x$ in $X_{s}$. Let

$$
X \stackrel{p}{\leftarrow} X \times_{S} X \stackrel{q}{\rightarrow} X
$$

denote the natural projections. The normalized Poincaré sheaf is then

$$
\mathcal{P}=\mathcal{I}_{\Delta}^{*} \otimes p^{*} \mathcal{O}_{X}\left(-S_{0}\right) \otimes q^{*} \mathcal{O}_{X}\left(-S_{0}\right) \otimes q^{*} \pi^{*} \omega_{S}
$$

where $\mathcal{I}_{\Delta}$ is the ideal sheaf of the diagonal $\Delta: X \rightarrow X \times_{S} X$.

\subsection{Fourier-Mukai transforms. Let}

$$
\phi_{\mathcal{K}}: D^{b} \operatorname{Coh}(X) \rightarrow D^{b} \operatorname{Coh}(X), \mathcal{E} \mapsto R q_{*}\left(p^{*} \mathcal{E} \stackrel{L}{\otimes} \mathcal{K}\right) .
$$

denote the Fourier-Mukai transform with kernel $\mathcal{K} \in D^{b} \operatorname{Coh}\left(X \times_{S} X\right)$.

We are mostly interested in the Fourier-Mukai transform $\phi_{\mathcal{P}}$ with kernel the Poincaré sheaf $\mathcal{P}$. We have the following facts.

Lemma 1. (1) The transform $\phi_{\mathcal{P}}$ is an auto-equivalence with inverse $\phi_{\mathcal{Q}}$ where $\mathcal{Q}:=\mathcal{P}^{\vee} \otimes p^{*} \pi^{*} \omega_{S}^{\vee}[1]$.

(2) Let inv : $X \rightarrow X$ be the involution which, under the identification $X \cong \hat{X}$, sends a torsion free sheaf to its dual. Then

$$
\phi_{\mathcal{P}} \circ \phi_{\mathcal{P}}=\operatorname{inv}^{*} \circ \mathbb{T}_{\pi^{*} \omega_{S}}[-1] .
$$

(3) Let $\mathbb{D}$ be the dual functor. Then

$$
\mathbb{D} \circ \phi_{\mathcal{P}}=\phi_{\mathcal{Q}} \circ \mathbb{D}=\phi_{\mathcal{P}}^{-1} \circ \mathbb{D} \text {. }
$$

Proof. For (1) see [6] or [2, Prop 2.5]. Then use $\phi_{\mathcal{P}^{*}}=$ inv $^{*} \circ \phi_{\mathcal{P}}$ to prove the second. The third follows by relative Grothendieck-Verdier duality applied to the morphism $q: X \times_{B} X \rightarrow X$, and using $\omega_{X \times_{S} X}=p^{*} \pi^{*} \omega_{S}^{\vee}$.

\section{Lemma 2.}


(1) $\phi_{\mathcal{P}}\left(\mathcal{O}_{X}\right)=\iota_{*} \omega_{S}[-1]$

(2) For every line bundle $\mathcal{M}$ on $S$, we have $\phi_{\mathcal{P}}\left(\iota_{*} \mathcal{M}\right)=\pi^{*} \mathcal{M}$.

(3) $\phi_{\mathcal{P}}\left(\mathcal{O}_{X}\left(S_{0}\right)\right)=\pi^{*} \omega_{S}\left(-S_{0}\right)$

(4) If $n>0$ then $\phi_{\mathcal{P}}\left(\mathcal{O}_{X}\left(-n S_{0}\right)\right)[1]$ is a locally free sheaf of rank $n$.

Proof. (1) We have $\mathcal{O}_{X}=\phi_{\mathcal{P}}\left(\mathcal{O}_{S_{0}}\right)$. Hence, by Lemma 1 (ii)

$$
\phi_{\mathcal{P}}\left(\mathcal{O}_{X}\right)=\phi_{\mathcal{P}}\left(\phi_{\mathcal{P}}\left(\mathcal{O}_{S_{0}}\right)\right)=\pi^{*} \omega_{S} \otimes \mathcal{O}_{S}[-1]=\iota_{*} \omega_{S}[-1] .
$$

(2) Since tensoring with a line bundle pulled back from $S$ commutes with $\phi_{\mathcal{P}}$, this follows from (1) by tensoring with $\pi^{*} \mathcal{M}$.

(3) The vanishing $H^{1}\left(X_{s}, \mathcal{O}_{X_{s}}\left(0_{s}\right)\right)=0$ for all $s \in S$ implies $\phi_{\mathcal{P}}\left(\mathcal{O}_{X}\left(S_{0}\right)\right)$ is concentrated in degree 0 . Applying $\phi_{\mathcal{P}}$ to the short exact sequence

$$
0 \rightarrow \mathcal{O}_{X} \rightarrow \mathcal{O}_{X}\left(S_{0}\right) \rightarrow \mathcal{O}_{S_{0}}\left(S_{0}\right)=\iota_{*} \omega_{S} \rightarrow 0
$$

and using (1) and (2) we obtain the exact sequence

$$
0 \rightarrow \phi_{\mathcal{P}}\left(\mathcal{O}_{X}\left(S_{0}\right)\right) \rightarrow \pi^{*} \omega_{S} \rightarrow \iota_{*} \omega_{S} \rightarrow 0 .
$$

(4) This follows either directly using the base change formula, or alternatively by induction over $n$ using the sequence

$$
0 \rightarrow \mathcal{O}_{X}\left(-n S_{0}\right) \rightarrow \mathcal{O}_{X}\left(-(n-1) S_{0}\right) \rightarrow \iota_{*} \omega_{S}^{-(n-1)} \rightarrow 0 .
$$

\subsection{Sheaves supported on fibers. Let}

$$
\alpha \in \operatorname{Pic}(S)
$$

be a ample divisor on $S$ such that $\alpha+K_{S}$ is ample. The induced divisor

$$
\lambda=\pi^{*} \alpha+S_{0} \in \operatorname{Pic}(X)
$$

is ample by an application of the Nakai-Moishezon criterion.

Consider the full subcategory of sheaves supported in dimension $\leq 1$,

$$
\operatorname{Coh}^{\leq 1}(X) \subset \operatorname{Coh}(X) \text {. }
$$

The slope of a sheaf $A \in \operatorname{Coh}^{\leq 1}(X)$ is defined by

$$
\mu(A)=\frac{\chi(A)}{\operatorname{ch}_{2}(A) \cdot \lambda} \in(-\infty, \infty]
$$

with the convention that 0 -dimensional sheaves have slope $+\infty$. A sheaf $B$ is stable (semistable) if $\mu(A)<(\leq) \mu(B)$ for every proper subsheaf $A \subset B$.

We say a sheaf $A \in \mathrm{Coh}^{\leq 1}(X)$ is supported on fibers of $\pi$ if the reduced support of $A$ is contained in the union of finitely many fibers $X_{s}, s \in S$. Let

$$
\mathcal{C} \subset \operatorname{Coh}^{\leq 1}(X)
$$

be the full subcategory of sheaves supported on fibers of $\pi$. The existence and uniqueness of Harder-Narasimhan filtrations in $\mathcal{C}$ with respect to $\mu$ is induced by the corresponding properties in $\mathrm{Coh}^{\leq 1}(X)$. 
For an interval $I \subset(-\infty, \infty]$ we let $\mathcal{C}_{I}$ denote the extension closure of all semistable sheaves in $\mathcal{C}$ with slope in $I$, together with the zero object,

$$
\mathcal{C}_{I}=\left\langle A \in \mathcal{C} \mid \begin{array}{c}
A \text { is } \mu \text {-semistable } \\
\text { with } \mu(A) \in I
\end{array}\right\rangle \cup\{0\} .
$$

The following proposition connects the notion of semi-stability to the Fourier-Mukai transform $\phi_{\mathcal{P}}$.

Proposition 2. Let $A \in \mathcal{C}$ be a semistable sheaf of slope $\mu_{0}=\mu(A)$. With the convention $-1 / \infty=0$ and $-1 / 0=\infty$ we have:

(1) If $\mu_{0} \in(0, \infty]$, then $\phi_{\mathcal{P}}(A)$ is a semistable sheaf in $\mathcal{C}$ of slope $-1 / \mu_{0}$.

(2) If $\mu_{0} \in(-\infty, 0]$, then $\phi_{\mathcal{P}}(A)=T[-1]$ for a semistable sheaf $T \in \mathcal{C}$ of slope $-1 / \mu_{0}$.

Proof. By taking a Jordan-Hölder filtration we may assume $A$ is stable and hence the pushforward of a stable sheaf from a fiber $X_{s}$. The claim then follows from [12, Thm 2.21] or along the lines of [2, Thm 3.2].

\section{2. $\pi$-STABLE PAIRS AND WALL-CROSSING}

2.1. Stable and $\pi$-stable pairs. A stable pair is the datum $(\mathcal{F}, s)$ of a pure 1-dimensional sheaf $\mathcal{F}$ and a section $s \in H^{0}(X, \mathcal{F})$ with 0 -dimensional cokernel. Following [32] we identify a stable pair $(\mathcal{F}, s)$ with the complex

$$
I^{\bullet}=\left[\mathcal{O}_{X} \stackrel{s}{\rightarrow} \mathcal{F}\right]
$$

in $D^{b} \operatorname{Coh}(X)$ where $\mathcal{O}_{X}$ sits in degree 0. A stable pair has class $\operatorname{ch}_{2}(\mathcal{F})=\beta$ and Euler characteristic $n$ precisely if $\operatorname{ch}\left(I^{\bullet}\right)=(1,0,-\beta,-n)$.

Recall the following characterization of stable pairs in [21], see also [38, Defn. 3.1].

Lemma 3. An object $I^{\bullet} \in D^{b} \operatorname{Coh}(X)$ with $\operatorname{ch}\left(I^{\bullet}\right)=(1,0,-\beta,-n)$ is a stable pair if and only if

(1) $h^{i}\left(I^{\bullet}\right)=0$ whenever $i \neq 0,1$.

(2) $h^{0}\left(I^{\bullet}\right)$ is torsion free and $h^{1}\left(I^{\bullet}\right)$ is 0-dimensional.

(3) $\operatorname{Hom}\left(Q[-1], I^{\bullet}\right)=0$ for every 0 -dimensional sheaf $Q$.

The definition of $\pi$-stable pairs is parallel to the characterization above but with 0-dimensional sheaves replaced by $\pi$-torsion sheaves which are defined as follows.

Definition 1. A sheaf $A \in \mathrm{Coh}^{\leq 1}(X)$ is $\pi$-torsion if the following conditions are satisfied:

(1) $A$ is supported on fibers of $\pi: X \rightarrow S$,

(2) $\phi_{\mathcal{P}}\left(A\left(S_{0}\right)\right)$ is a sheaf. 
We define $\pi$-stable pairs with respect to the elliptic fibration $\pi: X \rightarrow S$.

Definition 2. An object $I^{\bullet} \in D^{b} \operatorname{Coh}(X)$ with $\operatorname{ch}\left(I^{\bullet}\right)=(1,0,-\beta,-n)$ is a $\pi$-stable pair if

(1) $h^{i}\left(I^{\bullet}\right)=0$ whenever $i \neq 0,1$.

(2) $h^{0}\left(I^{\bullet}\right)$ is torsion free and $h^{1}\left(I^{\bullet}\right)$ is $\pi$-torsion.

(3) $\operatorname{Hom}\left(Q[-1], I^{\bullet}\right)=0$ for every $\pi$-torsion sheaf $Q$.

In [38, 4.2] Toda considered objects in the derived category which are characterized by the conditions of Lemma 3 but with 0-dimensional sheaves replaced by certain sheaves supported in dimension $\leq 1$. The associated invariants were termed $L$-invariants and play a central role in the proof of rationality of Pandharipande-Thomas invariants. Hence we may consider $\pi$ stable pairs to be a variant of the objects defining $L$-invariants. In particular, the definition of $\pi$-stable pair invariants and the proof of the wall-crossing formulas discussed below are parallel to the discussion in [38] and we will be brief.

2.2. Slope stability. Let $\mathcal{L}$ be a polarization on the 3 -fold $X$. The $\mathcal{L}$-slope of a coherent sheaf $\mathcal{E} \in \operatorname{Coh}(X)$ is

$$
\mu_{\mathcal{L}}(\mathcal{E})=\frac{c_{1}(\mathcal{E}) \cdot \mathcal{L}^{2}}{\operatorname{rank}(\mathcal{E})} \in \mathbb{Q} \cup\{\infty\}
$$

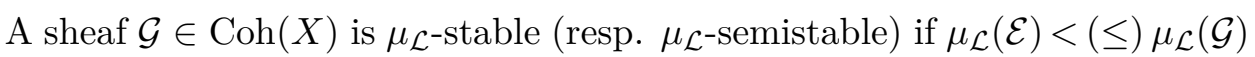
for every proper subsheaf $\mathcal{E} \subset \mathcal{G}$ of strictly smaller rank. For an interval $I \subset \mathbb{R} \cup\{\infty\}$ let

$$
\operatorname{Coh}_{I}(X)=\left\langle A \in \operatorname{Coh}(X) \mid \begin{array}{c}
A \text { is } \mu_{\mathcal{L}} \text {-semistable } \\
\text { with } \mu_{\mathcal{L}}(A) \in I
\end{array}\right\rangle \cup\{0\}
$$

be the extension closure of semistable sheaves of slope in $I$, together with the zero object. Let

$$
\mathcal{A}=\left\langle\mathrm{Coh}_{\leq 0}(X), \mathrm{Coh}_{>0}[-1]\right\rangle .
$$

be the tilt of $\operatorname{Coh}(X)$ along the torsion pair $\left(\mathrm{Coh}_{>0}(X), \mathrm{Coh}_{\leq 0}(X)\right)$. As in [38, 3.3] we will work inside the full abelian subcategory

$$
\mathcal{B}=\left\langle\operatorname{Coh}_{0}(X), \operatorname{Coh}^{\leq 1}(X)[-1]\right\rangle \subset \mathcal{A} .
$$

2.3. $\pi$-stable pair invariants. Let $\mathcal{M}$ be the moduli stack of objects in $\mathcal{A}$ with fixed Chern character $(1,0,-\beta,-n)$ and let

$$
\mathcal{P}_{n}^{\pi}(X, \beta) \subset \mathcal{M}
$$

be the substack of $\pi$-stable pairs with $\operatorname{ch}\left(I^{\bullet}\right)=(1,0,-\beta,-n)$.

Completely parallel to the case of $L$-invariants in [38, 4.2] we have the following Lemma. 
Lemma 4. The $\mathbb{C}$-valued points of $\mathcal{P}_{n}^{\pi}(X, \beta)$ form a constructible subset of $\mathcal{M}$, and $\operatorname{Aut}\left(I^{\bullet}\right)=\mathbb{C}^{*}$ for every $\pi$-stable pair $I^{\bullet}$.

We define the $\pi$-stable pair invariant

$$
\mathrm{P}_{n, \beta}^{\pi} \in \mathbb{Z}
$$

by taking the motive defined by the inclusion (8), multiplying by the motive $\left[\mathbb{C}^{*}\right]$ and applying the integration map of the motivic hall algebra of $\mathcal{A}$, compare [38, 4.2]. By Lemma 4 the invariant $\mathrm{P}_{n, \beta}^{\pi}$ is well-defined.

2.4. Wall-crossing. We follow closely the discussion in [38, 3.7]. Let

$$
\operatorname{Coh}_{\pi}(X) \subset D^{b} \operatorname{Coh}(X) \text { and } \operatorname{Coh}_{P}(X) \subset D^{b} \operatorname{Coh}(X)
$$

be the full categories of $\pi$-stable pairs and stable pairs respectively, and recall from (17) the category $\mathcal{C}_{I}$ of fiber sheaves defined by the interval $I \subset \mathbb{R} \cup\{\infty\}$.

By Proposition 2]a sheaf $A$ is $\pi$-torsion precisely if it is an element of $\mathcal{C}$ and all its Harder-Narasimhan factors have slope $\mu>-1$. Hence an argument identical to the proof of [38, Lem. 3.16] shows the following Lemma.

Lemma 5. We have the following identity in $\mathcal{B}$ :

$$
\left\langle\operatorname{Coh}_{P}(X), \mathcal{C}_{(-1, \infty)}[-1]\right\rangle=\left\langle\mathcal{C}_{(-1, \infty)}[-1], \operatorname{Coh}_{\pi}(X)\right\rangle .
$$

Define the generating series of $\pi$-stable pair invariants

$$
\mathrm{PT}_{H}^{\pi}(q, t)=\sum_{d \geq 0} \sum_{n \in \mathbb{Z}} \mathrm{P}_{n, H+d F}^{\pi} q^{n} t^{d}
$$

and let

$$
f(q, t)=\prod_{\ell, m \geq 1}\left(1-(-q)^{\ell} t^{m}\right)^{-\ell \cdot e(X)} .
$$

The main result of this section is the following $\mathrm{PT} / \pi$-PT correspondence.

Proposition 3. For every effective $H \in \operatorname{Pic}(S)$, we have

$$
\mathrm{PT}_{H}(q, t)=f\left(t q^{-1}, t\right)^{-1} f(q, t) \cdot \mathrm{PT}_{H}^{\pi}(q, t) .
$$

Proof. Consider the generalized Donaldson-Thomas invariant

$$
N_{n, \beta} \in \mathbb{Q}
$$

counting semistable sheaves of Chern character $(0,0, \beta, n)$, see [38, 3.6].

By applying the integration map of the motivic Hall algebra of $\mathcal{A}$ to the identity in Lemma 5 (see [38, Thm 3.17] for details) we obtain

$$
\mathrm{PT}_{H}(q, t)=\exp \left(\sum_{\substack{d \geq 0, n \in \mathbb{Z} \\ n / d \in(-1, \infty)}}(-1)^{n-1} n \mathrm{~N}_{n, d F} q^{n} t^{d}\right) \cdot \mathrm{PT}_{H}^{\pi}(q, t)
$$


The first factor on the right-hand side is the contribution of $\mathcal{C}_{(-1, \infty)}[-1]$. By [36. Thm 6.9] we have for all $d>0$

$$
\mathrm{N}_{n, d F}=\sum_{k \mid(n, d)} \frac{-e(X)}{k^{2}}
$$

We find

$$
\exp \left(\sum_{\substack{d>0, n \in \mathbb{Z} \\ n / d \in(0, \infty)}}(-1)^{n-1} n \mathrm{~N}_{n, d F} q^{n} t^{d}\right)=\prod_{\ell, m \geq 1}\left(1-(-q)^{\ell} t^{m}\right)^{-\ell e(X)}=f(q, t)
$$

and

$$
\exp \left(\sum_{\substack{d>0, n \in \mathbb{Z} \\ n / d \in(-1,0)}}(-1)^{n-1} n \mathrm{~N}_{n, d F} q^{n} t^{d}\right)=f\left(q^{-1} t, t\right)^{-1} .
$$

Plugging into (9) the proof is complete.

\section{The Derived equivalence $\Phi_{H}$}

3.1. Overview. In this Section we use the derived equivalence $\Phi_{H}$ to prove the following Theorem.

Theorem 7. Let $H \in \operatorname{Pic}(S)$ be effective and reduced. Then

$$
\mathrm{P}_{n, H+d F}=\mathrm{P}_{-n-2 h+2, H+(d+n+h-1) F}^{\pi}
$$

We immediately deduce Theorem 1 as a corollary.

Proof of Theorem [1. Rewriting Theorem 7 in generating series we find

$$
\mathrm{PT}_{H}(q, t)=t^{h-1} q^{-2(h-1)} \mathrm{PT}_{H}^{\pi}\left(q^{-1} t, t\right) .
$$

By applying Proposition 3 to the last term yields

$$
\mathrm{PT}_{H}(q, t)=t^{h-1} q^{-2(h-1)} \frac{f(q, t)}{f\left(q^{-1} t, t\right)} \cdot \mathrm{PT}_{H}\left(q^{-1} t, t\right) .
$$

By (11) we have

$$
\frac{f(q, t)}{f\left(q^{-1} t, t\right)}=\frac{\mathrm{PT}_{0}(q, t)}{\mathrm{PT}_{0}\left(q^{-1} t, t\right)}
$$

which completes the proof.

\subsection{Basic properties of $\Phi_{H}$. Let $H \in \operatorname{Pic}(S)$ be an effective class.}

Definition 3. Define the autoequivalence

$$
\Phi_{H}=\mathbb{D} \circ \mathbb{T}_{\pi^{*} \mathcal{O}_{S}\left(H-K_{S}\right)} \circ \mathbb{T}_{\mathcal{O}_{X}\left(S_{0}\right)} \circ \phi_{\mathcal{P}} \circ \mathbb{T}_{\mathcal{O}_{X}\left(S_{0}\right)} .
$$

For an element $\mathcal{E} \in D^{b} \operatorname{Coh}(X)$ we write

$$
\widetilde{\mathcal{E}}=\Phi_{H}(\mathcal{E}) .
$$


Definition 3 agrees with the definition of $\Phi_{H}$ in Section 0.10 , but is more convenient for computations since we work with the normalized Poincaré sheaf $\mathcal{P}$. We have several basic Lemmas.

Lemma 6. For all $\mathcal{E}, \mathcal{F} \in D^{b} \operatorname{Coh}(X)$

(1) $\widetilde{\mathcal{E}[i]}=\widetilde{\mathcal{E}}[-i]$.

(2) $\operatorname{Hom}(\mathcal{E}, \mathcal{F}) \cong \operatorname{Hom}(\widetilde{\mathcal{F}}, \widetilde{\mathcal{E}})$.

We show $\Phi_{H}$ acts on $D^{b} \operatorname{Coh}(X)$ as an involution.

Lemma 7. $\Phi_{H} \circ \Phi_{H}=\mathrm{id}_{D^{b} \operatorname{Coh}(X)}$.

Proof. By Lemma 1 we have $\mathbb{D} \circ \phi_{\mathcal{P}}=\phi_{\mathcal{P}}^{-1} \circ \mathbb{D}$. Hence, with

$$
\mathcal{L}:=O_{X}\left(S_{0}+\pi^{*} H-\pi^{*} K_{S}\right)
$$

and since tensoring with a line bundle pulled back from $S$ commutes with $\phi_{\mathcal{P}}$ we have

$$
\Phi_{H}^{2}=\left(\mathbb{D} \circ \mathbb{T}_{O_{X}\left(S_{0}\right)} \circ \phi_{\mathcal{P}} \circ \mathbb{T}_{\mathcal{L}}\right) \circ\left(\mathbb{D} \circ \mathbb{T}_{\mathcal{L}} \circ \phi_{\mathcal{P}} \circ \mathbb{T}_{O_{X}\left(S_{0}\right)}\right)=\operatorname{id}_{D^{b} \operatorname{Coh}(X)} .
$$

\section{Lemma 8.}

(1) $\widetilde{\mathcal{O}_{X}}=\mathcal{O}_{X}\left(-\pi^{*} H\right)$

(2) Let $x \in X$ be a point, let $\imath_{s}: X_{s} \hookrightarrow X$ be the inclusion, and let $\mathfrak{m}_{x}$ be the ideal sheaf of $x$ in $X_{s}$. Then

$$
\widetilde{\mathbb{C}_{x}}=\imath_{s *} \mathfrak{m}_{x}[-2] .
$$

(3) For all $n>1$ we have

$$
\Phi_{H}\left(\mathcal{O}_{X}\left(-n S_{0}\right)\right)=\mathcal{V}_{n}[1]
$$

for a locally free sheaf $\mathcal{V}_{n}$ of rank $n-1$.

Proof. (1) and (3) follow directly from Lemma 2 and the definition. For (2) we have

$$
\mathbb{T}_{\mathcal{O}_{X}\left(\pi^{*}\left(H-K_{S}\right)+S_{0}\right)} \circ \phi_{\mathcal{P}} \circ \mathbb{T}_{\mathcal{O}_{X}\left(S_{0}\right)}\left(\mathbb{C}_{x}\right)=\imath_{s *} \mathfrak{m}_{x}^{*}
$$

Hence using relative duality and since $\mathfrak{m}_{x}$ is reflexive on $X_{s}$ we get

$$
\widetilde{\mathbb{C}_{x}}=\mathbb{D}\left(\imath_{s *} \mathfrak{m}_{x}^{*}\right)=\imath_{s *}\left(\mathfrak{m}_{x}^{* \vee}\right)[-2]=\imath_{s *}\left(\mathfrak{m}_{x}\right)[-2] .
$$

We prove $\Phi_{H}$ acts as expected on the level of cohomology.

Lemma 9. Assume $\mathcal{E} \in D^{b} \operatorname{Coh}(X)$ has Chern character

$$
\operatorname{ch}(\mathcal{E})=(1,0,-(H+d F),-n),
$$

in $H^{2 *}(X, \mathbb{Q})=\sum_{i} H^{2 i}(X, \mathbb{Q})$, then

$$
\operatorname{ch}\left(\Phi_{H}(\mathcal{E})\right)=(1,0,-(H+(n+d+h-1) F), n+2 h-2) .
$$


Proof. By a direct calculation as in [1, 5.2], or by calculating $\widetilde{\mathcal{O}_{X}}, \widetilde{\mathcal{O}_{X_{s}}}, \widetilde{\mathbb{C}_{x}}$, and $\widetilde{\mathcal{O}_{\iota(B)}}$ with $B \in|H|$ directly as in Lemma 8 ,

We consider the action of $\Phi_{H}$ on the category $\mathcal{C}$ of 1-dimensional sheaves supported on fibers of $\pi$.

Lemma 10. Let $A \in \mathcal{C}$ be a semistable sheaf and let $\mu_{0}=\mu(A)$ be its slope. With the conventions $1 / 0=\infty$ and $\infty / \infty=1$ we have:

- If $\mu_{0} \in[-1, \infty]$, then $\widetilde{A}=G[-2]$ for a semistable sheaf $G \in \mathcal{C}$ of slope $-\mu_{0} /\left(1+\mu_{0}\right) \geq-1$.

- If $\mu_{0} \in(-\infty,-1)$, then $\widetilde{A}=G[-1]$ for a semistable sheaf $G \in \mathcal{C}$ of slope $-\mu_{0} /\left(1+\mu_{0}\right)<-1$.

In particular, for an interval $[a, b] \subset[-1, \infty]$ we have

$$
\Phi_{H}\left(\mathcal{C}_{[a, b]}\right)=\mathcal{C}_{[-b /(1+b),-a /(1+a)]}[-2]
$$

and likewise for open or half-open intervals in $[-1, \infty]$.

Proof. This follows from Proposition 2 and the following: If $A \in \mathcal{C}$ is semistable of slope $a<\infty$, then $\mathbb{D}(A)=T[-2]$ for a semistable sheaf $T \in \mathcal{C}$ of slope $-a$.

Lemma 11. Let $\mathcal{I}_{C}$ be the ideal sheaf of a Cohen-Macaulay curve $C \subset X$ with no components supported on fibers of $\pi$. Then $\widetilde{\mathcal{I}_{C}}$ is a sheaf.

Proof. For all $x \in X$ and $i \geq 1$ we claim the vanishing of

$$
\operatorname{Hom}\left(\widetilde{\mathcal{I}_{C}}, \mathbb{C}_{x}[-i]\right)=\operatorname{Hom}\left(\imath_{s *} \mathfrak{m}_{x}, \mathcal{I}_{C}[2-i]\right)
$$

with $s=\pi(x)$ and $\imath_{s}: X_{s} \hookrightarrow X$. The case $i \geq 3$ is immediate. The case $i=2$ follows since $\mathcal{I}_{C}$ is torsion free. For $i=1$ we have

$$
\operatorname{Ext}^{1}\left(\imath_{s *} \mathfrak{m}_{x}, \mathcal{I}_{C}\right)=\operatorname{Hom}\left(\imath_{s *} \mathfrak{m}_{x}, \mathcal{O}_{C}\right)=\operatorname{Hom}_{C}\left(j^{*} \imath_{s *} \mathfrak{m}_{x}, \mathcal{O}_{C}\right)
$$

where $j: C \rightarrow X$ is the inclusion. Since $C$ has no components supported on fibers, $j^{*} \imath_{s *} \mathfrak{m}_{x}$ is zero-dimensional. Since $C$ is Cohen-Macaulay, $\mathcal{O}_{C}$ is pure and we conclude $\operatorname{Hom}_{C}\left(j^{*} \imath_{s *} \mathfrak{m}_{x}, \mathcal{O}_{C}\right)=0$. We conclude $\widetilde{\mathcal{I}_{C}}$ is concentreated in degrees $\leq 0$.

We prove the lower bound. By applying $\Phi_{H}$ to the exact sequence

$$
0 \rightarrow \mathcal{I}_{C} \rightarrow \mathcal{O}_{X} \rightarrow \mathcal{O}_{C} \rightarrow 0
$$

and taking the long exact sequence in cohomology it suffices to prove $\widetilde{\mathcal{O}_{C}}$ is concentrated in degrees $\geq 1$.

For all $\mathcal{L} \in \operatorname{Pic}(S), n>1$ and $i \leq 0$ we have by Lemma 8

$$
\operatorname{Hom}\left(\pi^{*} \mathcal{L}^{\vee}\left(-n S_{0}\right), \widetilde{\mathcal{O}_{C}}[i]\right)=\operatorname{Hom}\left(\mathcal{O}_{C}, \pi^{*} \mathcal{L} \otimes \mathcal{V}_{n}[i+1]\right)=0,
$$

since $\mathcal{O}_{C}$ is supported on a curve and $\mathcal{V}_{n}$ is locally free. Since $\pi^{*} \mathcal{L}\left(n S_{0}\right)$ can be taken arbitrarily positive here, $\widetilde{\mathcal{O}_{C}}$ is concentrated in degrees $\geq 1$. 
3.3. Two term complexes: the reduced case. Consider the following conditions on complexes $I^{\bullet} \in D^{b} \operatorname{Coh}(X)$ :

(a) $\operatorname{ch}\left(I^{\bullet}\right)=(1,0,-(H+d F),-n)$ for some $d \geq 0, n \in \mathbb{Z}$.

(b) $I^{\bullet}$ is two-term: $h^{i}\left(I^{\bullet}\right)=0$ whenever $i \neq 0,1$.

(c) $h^{0}\left(I^{\bullet}\right)$ is torsion free.

(d) $h^{1}\left(I^{\bullet}\right) \in \mathcal{C}_{[-1, \infty]}$.

The aim of this subsection is to prove the following proposition.

Proposition 4. Let $H \in \operatorname{Pic}(S)$ be a reduced effective class. Then a complex $I^{\bullet} \in D^{b} \operatorname{Coh}(X)$ satisfies Properties (a-d) above if and only if $\widetilde{I^{\bullet}}$ does.

First we prove a lemma.

Lemma 12. Let $D$ be an effective divisor on $S$, consider the surface

$$
W=\pi^{-1}(D)
$$

and let $\mathcal{I}_{C / W}$ be the ideal sheaf of a curve $C$ in $W$. Assume the projection $C \rightarrow D$ is an isomorphism over a dense open subset $U \subset D$, and the class of $C$ is $\iota_{*} D+m F \in H_{2}(X, \mathbb{Z})$ for some $m \in \mathbb{Z}$. Then

$$
\widetilde{\mathcal{I}_{C / W}}=\mathcal{E}[-1]
$$

for a 1-dimensional sheaf $\mathcal{E}$.

Proof. The proof proceeds in three steps.

Step 1. Since $\mathcal{I}_{C / W}\left(S_{0}\right)$ is a sheaf and $q: X \times_{S} X \rightarrow X$ has relative dimension 1, the complex

$$
A=\phi_{\mathcal{P}}\left(\mathcal{I}_{C / W}\left(S_{0}\right)\right)
$$

is concentrated in degrees 0 and 1 . We show both $h^{0}(A)$ and $h^{1}(A)$ are of dimension $\leq 1$. Since $C \rightarrow D$ is an isomorphism away from a 0 -dimensional subset of $D$ we have

$$
\operatorname{ch}\left(\mathcal{I}_{C / W}\right)=\left(0, \pi^{*} D,-\iota_{*} D+a F, b\right)
$$

for some $a, b \in \mathbb{Z}$. By a direct calculation this yields

$$
\operatorname{ch}(A)=\left(0,0,-\iota_{*} D+a^{\prime} F, b^{\prime}\right)
$$

for some $a^{\prime}, b^{\prime}$. Hence it suffices to show $h^{1}(A)$ is supported on curves.

Let $U \subset D$ be the open subset over which $C \rightarrow D$ is an isomorphism. In particular $\left.\mathcal{O}_{C}\right|_{\pi^{-1}(U)}$ is flat over $\left.\mathcal{O}_{D}\right|_{U}$. For every $y \in X$ with $s=\pi(y) \in U$ we have by base change

$$
h^{1}(A) \otimes \mathbb{C}_{y}=H^{1}\left(X_{s},\left.\mathcal{I}_{C / W}\left(S_{0}\right)\right|_{X_{s}} \otimes \mathcal{P}_{y}\right)
$$


where $\mathcal{P}_{y}=\phi_{\mathcal{P}}\left(\mathbb{C}_{y}\right)$ is the sheaf corresponding to the point $y$. Applying flatness we obtain

$$
h^{1}(A) \otimes \mathbb{C}_{y}=H^{1}\left(X_{s}, \mathcal{I}_{C \cap X_{s} / X_{s}}\left(S_{0}\right) \otimes \mathcal{P}_{y}\right)
$$

which is non-zero only if $y=C \cap X_{s}$. We conclude

$$
\operatorname{Supp}\left(h^{1}(A)\right) \cap \pi^{-1}(U)=C \cap \pi^{-1}(U),
$$

and therefore that $h^{1}(A)$ is 1-dimensional.

Step 2. We prove $\widetilde{I_{C / W}}$ is concentrated in degrees $\geq 1$.

Let $\mathcal{L}=\mathcal{O}_{X}\left(S_{0}+\pi^{*}\left(H-K_{S}\right)\right)$ and let $G_{i}:=h^{i}(A) \otimes \mathcal{L}$ for $i=0,1$. Applying $\mathbb{D} \circ \mathbb{T}_{\mathcal{L}}$ to the canonical exact triangle

$$
h^{0}(A) \rightarrow A \rightarrow h^{1}(A)[-1]
$$

yields the exact triangle

$$
\mathbb{D}\left(G_{1}\right)[1] \rightarrow \widetilde{\mathcal{I}_{C / W}} \rightarrow \mathbb{D}\left(G_{0}\right) .
$$

By Step 1 the sheaves $G_{i}$ are of dimension $\leq 1$. Hence the $\mathbb{D}\left(G_{i}\right)$ are concentrated in degrees $\geq 2$, and so $\widetilde{\mathcal{I}_{C / W}}$ is concentrated in degrees $\geq 1$.

Step 3. We show $\widetilde{\mathcal{I}_{C / W}}$ is concentrated in degrees $\leq 1$.

By Lemma 8 we have for all $x \in X$

$$
\operatorname{Hom}\left(\widetilde{\mathcal{I}_{C / W}}, \mathbb{C}_{x}[-i]\right)=\operatorname{Hom}\left(\imath_{s *} \mathfrak{m}_{x}, \mathcal{I}_{C / W}[2-i]\right),
$$

which we claim vanishes for all $i \geq 2$. The case $i \geq 3$ is immediate. For the vanishing in case $i=2$, we apply $\operatorname{Hom}\left(l_{s *} \mathfrak{m}_{x}, \cdot\right)$ to the exact sequence

$$
0 \rightarrow \mathcal{O}_{X}\left(-\pi^{*} D\right) \rightarrow \mathcal{I}_{C} \rightarrow \mathcal{I}_{C / W} \rightarrow 0
$$

and use that $\imath_{s *} \mathfrak{m}_{x}$ is supported in dimension 1 and $\mathcal{I}_{C}$ is torsion free.

3.4. Proof of Proposition 4. Assume a complex $I^{\bullet}$ satisfies (a-d). Since $\Phi_{H}$ is an involution it is enough to prove $\widetilde{I^{\bullet}}$ satisfies (a-d).

By Lemma 9 the complex $\widetilde{I^{\bullet}}$ satisfies (a), so we need to show (b,c,d). We will make several reduction steps.

Step 1. Since $h^{1}\left(I^{\bullet}\right) \in \mathcal{C}_{[-1, \infty]}$ we have $\widetilde{h^{1}\left(I^{\bullet}\right)}=G[-2]$ for some $G \in$ $\mathcal{C}_{[-1, \infty]}$. Applying $\Phi_{H}$ to the canonical exact triangle

$$
h^{0}\left(I^{\bullet}\right) \rightarrow I^{\bullet} \rightarrow h^{1}\left(I^{\bullet}\right)[-1]
$$

and taking the long exact sequence in cohomology we therefore find

$$
0 \rightarrow h^{0}\left(\widetilde{I^{\bullet}}\right) \rightarrow h^{0}\left(\widetilde{h^{0}\left(I^{\bullet}\right)}\right) \rightarrow G \rightarrow h^{1}\left(\widetilde{I^{\bullet}}\right) \rightarrow h^{1}\left(\widetilde{h^{0}\left(I^{\bullet}\right)}\right) \rightarrow 0
$$

and $h^{i}\left(\widetilde{I^{\bullet}}\right)=h^{i}\left(\widetilde{h^{0}\left(I^{\bullet}\right)}\right)$ for $i \neq 0,1$. Hence $\widetilde{I^{\bullet}}$ satisfies (b-d) if $\widetilde{h^{0}\left(I^{\bullet}\right)}$ does. We may therefore assume that $I^{\bullet}$ is the ideal sheaf $\mathcal{I}_{C}$ for some curve $C \subset X$. 
Step 2. Let $C^{\prime} \subset C$ be the unique maximal Cohen-Macaulay subcurve. We have the sequence

$$
0 \rightarrow \mathcal{I}_{C} \rightarrow \mathcal{I}_{C^{\prime}} \rightarrow Q \rightarrow 0
$$

where $Q$ is 0 -dimensional. Since $\widetilde{Q}=G[-2]$ for some $G \in \mathcal{C}_{-1}$ we have the exact sequence

$$
0 \rightarrow h^{1}\left(\widetilde{\mathcal{I}_{C^{\prime}}}\right) \rightarrow h^{1}\left(\widetilde{\mathcal{I}_{C}}\right) \rightarrow G \rightarrow 0 .
$$

and $h^{i}\left(\widetilde{\mathcal{I}_{C^{\prime}}}\right)=h^{i}\left(\widetilde{\mathcal{I}_{C}}\right)$ for all $i \neq 1$. Hence $\widetilde{\mathcal{I}_{C}}$ satisfies (b-d) if $\widetilde{\mathcal{I}_{C^{\prime}}}$ does, and so we may assume $C$ is Cohen-Macaulay.

Step 3. Let $D \subset S$ be the divisor on $S$ defined by the image $\pi(C) \subset S$. (In particular, $D$ remembers only the codimension 1 locus of $\pi(C)$ and not isolated points or thickening at points.) We consider the curve

$$
C^{\prime}=C \cap \pi^{-1}(D) \subset \pi^{-1}(D) .
$$

Since $C^{\prime} \subset C$, we have the exact sequence

$$
0 \rightarrow K \rightarrow \mathcal{O}_{C} \rightarrow \mathcal{O}_{C^{\prime}} \rightarrow 0 .
$$

with $K \in \mathcal{C}$.

Claim: $K$ is in $\mathcal{C}_{[0, \infty)}$.

Proof of Claim. By construction $K$ is supported on fibers of $\pi$. Moreover since $C$ is Cohen-Macaulay (which is equivalent to $\mathcal{O}_{C}$ is pure) $K$ is pure of dimension 1.

For our convenience let us assume $K$ is supported over a point $s \in S$.

If $s \notin D$ then $K$ is the structure sheaf of the connected component of $C$ that lies over $s \in S$. It follows that $K$ admits a surjection $\mathcal{O}_{X} \rightarrow K$. Hence, if $K^{\prime}$ is the the Harder-Narasimhan factor of $K$ with smallest slope $\mu_{0}$, then the natural composition $\mathcal{O}_{X} \rightarrow K \rightarrow K^{\prime}$ is non-zero. Therefore $\mu_{0} \geq 0$ by semistability, and so $K \in \mathcal{C}_{[0, \infty]}$, see also the proof of [36, Prop 6.8].

If $s \in D$ then $K$ is supported over $F_{n}=\operatorname{Spec} \mathcal{O}_{X} / \pi^{*} \mathfrak{m}_{s}^{n}$ for some $n \gg 0$ where $\mathfrak{m}_{s}$ is the ideal sheaf of $s \in S$. Let $f \in \mathcal{O}_{S}$ be the local equation of $D$ near $s$, and let $\bar{f}$ be its image in $\mathcal{O}_{S} / \mathfrak{m}_{s}^{n}$. Consider the exact sequence

$$
0 \rightarrow(\bar{f}) \rightarrow \mathcal{O}_{S} / \mathfrak{m}_{s}^{n} \rightarrow \mathcal{O}_{S} /\left(\mathfrak{m}_{s}^{n}, f\right) \rightarrow 0 .
$$

By flatness of $\pi$, (11) remains exact under pullback:

$$
0 \rightarrow \pi^{*}(\bar{f}) \rightarrow \mathcal{O}_{X} / \pi^{*} \mathfrak{m}_{s}^{n} \rightarrow \mathcal{O}_{X} / \pi^{*}\left(\mathfrak{m}_{s}^{n}, f\right) \rightarrow 0 .
$$

Tensoring (12) with $\mathcal{O}_{C}$ we obtain

$$
\pi^{*}(\bar{f}) \otimes_{\mathcal{O}_{X}} \mathcal{O}_{C} \rightarrow K \hookrightarrow \mathcal{O}_{C \cap F_{n}} \rightarrow \mathcal{O}_{C^{\prime} \cap F_{n}} \rightarrow 0 .
$$

For every zero-dimensional sheaf $T$ on $S$ a surjection $\mathcal{O}_{S}^{k} \rightarrow T \rightarrow 0$ for some $k \geq 1$ induces a surjection $\mathcal{O}_{X}^{k} \rightarrow \mathcal{O}_{C}^{k} \rightarrow \pi^{*} T \otimes_{\mathcal{O}_{X}} \mathcal{O}_{C}$ (by pullback via $\pi^{*}$ 
and tensoring with $\left.\mathcal{O}_{C}\right)$. Applying this to $T=(\bar{f})$ we obtain a composition of surjections

$$
\mathcal{O}_{X}^{k} \rightarrow \pi^{*}(\bar{f}) \otimes \mathcal{O}_{X} \mathcal{O}_{C} \rightarrow K
$$

Let again $K^{\prime}$ be the Harder-Narasimhan factor of $K$ with smallest slope. Then composing (13) with $K \rightarrow K^{\prime}$ yields a non-zero section of $K^{\prime}$. We find $K^{\prime} \in \mathcal{C}_{[0, \infty)}$ and hence $K \in \mathcal{C}_{[0, \infty)}$.

We return to Step 3 of the reduction. From (10) we obtain

$$
0 \rightarrow \mathcal{I}_{C} \rightarrow \mathcal{I}_{C^{\prime}} \rightarrow K \rightarrow 0 .
$$

Since $K \in \mathcal{C}_{[0, \infty]}$ we have $\widetilde{K}=A[-2]$ for some $A \in \mathcal{C}_{(-1, \infty)}$. Hence argueing as in Step 2 we find $\widetilde{\mathcal{I}_{C}}$ satisfies (b-d) if $\widetilde{\mathcal{I}_{C^{\prime}}}$ does. Hence we may assume $C$ is contained inside the surface $\pi^{-1}(D)$.

Step 4. We prove Properties (b) and (d).

Let $C^{\prime}$ be the union of all irreducible component of $C$ which are not supported on fibers of $\pi$. The canonical exact sequence

$$
0 \rightarrow \mathcal{I}_{C} \rightarrow \mathcal{I}_{C^{\prime}} \rightarrow K \rightarrow 0
$$

with $K \in \mathcal{C}$ yields the exact triangle

$$
\widetilde{K} \rightarrow \widetilde{\mathcal{I}_{C^{\prime}}} \rightarrow \widetilde{\mathcal{I}_{C}} .
$$

By Lemma 11 the complex $\widetilde{\mathcal{I}_{C^{\prime}}}$ is a sheaf. Hence taking long exact sequence of (14) yields $h^{i}\left(\widetilde{\mathcal{I}_{C}}\right)=0$ for $i \neq 0,1$ (Property (b)), and the isomorphism

$$
h^{1}\left(\widetilde{\mathcal{I}_{C}}\right) \stackrel{\simeq}{\longrightarrow} h^{2}(\widetilde{K}) .
$$

Let $0 \rightarrow K_{+} \rightarrow K \rightarrow K_{-} \rightarrow 0$ be the unique filtration of $K$ with $K_{+} \in$ $\mathcal{C}_{[-1, \infty]}$ and $K_{-} \in \mathcal{C}_{[-\infty,-1)}$. Then applying $\Phi_{H}$, using the long exact sequence in cohomology and Lemma 10 yields

$$
h^{2}(\widetilde{K})=h^{2}\left(\widetilde{K_{+}}\right) \in \mathcal{C}_{[-1, \infty]}
$$

Step 5. We prove that $h^{0}\left(\widetilde{I_{C}}\right)$ is an ideal sheaf, which implies Property (c). Let $D$ be the divisor on $S$ as in Step 3 and recall that we may assume

$$
C \subset W=\pi^{-1}(D) .
$$

Since the class $H \in \operatorname{Pic}(S)$ is reduced we have $D \in|H|$. Hence the inclusion (15) gives the exact sequence

$$
0 \rightarrow \mathcal{O}_{X}\left(-\pi^{*} H\right) \rightarrow \mathcal{I}_{C} \rightarrow \mathcal{I}_{C / W} \rightarrow 0
$$

where $\mathcal{I}_{C / W}$ is the ideal sheaf of $C$ in $W$. Applying $\Phi_{H}$ and taking cohomology yields

$$
0 \rightarrow h^{0}\left(\widetilde{\mathcal{I}_{C / W}}\right) \rightarrow h^{0}\left(\widetilde{\mathcal{I}_{C}}\right) \rightarrow \mathcal{O}_{X} \rightarrow h^{1}\left(\widetilde{\mathcal{I}_{C / W}}\right) \rightarrow h^{1}\left(\widetilde{\mathcal{I}_{C}}\right) \rightarrow 0 .
$$


Since $C$ has class $H+d F$ and $H$ is reduced, the projection $C \rightarrow D$ is generically of degree 1 on every component of $C$. By Lemma 12 the complex $\widetilde{\mathcal{I}_{C / W}}[1]$ is a sheaf. Hence $h^{0}\left(\widetilde{\mathcal{I}_{C}}\right)$ is an ideal sheaf.

The proof of Proposition 4 requires $H$ to be reduced only in Step 5 . Hence we obtain the following corollary in case $H$ is not necessarily reduced.

Corollary 2. Let $H \in \operatorname{Pic}(S)$ be effective. If $I^{\bullet} \in D^{b} \operatorname{Coh}(X)$ satisfies properties (a-d) above, then $\widetilde{I^{\bullet}}$ satisfies (a), (b) and (d).

3.5. Purity. Let $I^{\bullet} \in D^{b} \operatorname{Coh}(X)$ be a complex which satisfies conditions (a-d) of Section 3.3 .

Lemma 13. Let $a \in[-1, \infty]$. Then the following are equivalent:

- $\operatorname{Hom}\left(Q[-1], I^{\bullet}\right)=0$ for all $Q \in \mathcal{C}_{(a, \infty]}$, and

- $h^{1}\left(\widetilde{I^{\bullet}}\right) \in \mathcal{C}_{[-a /(1+a), \infty]}$.

The same holds when the intervals are replaced by $[a, \infty]$ and $(-1 /(1+a), \infty]$ respectively.

Proof. By Lemma 10 we have

$$
\operatorname{Hom}\left(Q[-1], I^{\bullet}\right)=0
$$

for all $Q \in \mathcal{C}_{(a, \infty]}$ if and only if

$$
\operatorname{Hom}\left(\widetilde{I^{\bullet}}, G[-1]\right)=\operatorname{Hom}\left(h^{1}\left(\widetilde{I^{\bullet}}\right), G\right)=0
$$

for all $G \in \mathcal{C}_{[-1,-a /(1+a))}$. Since by Corollary 2 we have $h^{1}\left(\widetilde{I^{\bullet}}\right) \in \mathcal{C}_{[-1, \infty]}$, this is equivalent to

$$
h^{1}\left(\widetilde{I^{\bullet}}\right) \in \mathcal{C}_{[-a /(1+a), \infty]} .
$$

The last claim of the Lemma follows by a parallel argument.

We obtain the following corollary which implies Theorem 7.

Corollary 3. Let $H \in \operatorname{Pic}(S)$ be reduced. Then a complex $I^{\bullet}$ is a stable pair if and only if $\widetilde{I^{\bullet}}$ is a $\pi$-stable pair.

Proof. Let $I^{\bullet} \in D^{b} \operatorname{Coh}(X)$. By Proposition 4 and by Lemma 13 with $a=-1$ and $a=\infty$ respectively we obtain:

(1) The complex $I^{\bullet}$ satisfies (a-d) (in Section 3.3) if and only if $\widetilde{I^{\bullet}}$ does.

(2) Suppose $I^{\bullet}$ satisfies (a-d), then $h^{1}\left(I^{\bullet}\right)$ is 0-dimensional if and only if

$$
\operatorname{Hom}\left(Q[-1], \widetilde{I^{\bullet}}\right)=0
$$

for all $\pi$-torsion sheaves $Q$. 
(3) Suppose $I^{\bullet}$ satisfies (a-d), then $h^{1}\left(I^{\bullet}\right)$ is $\pi$-torsion if and only if

$$
\operatorname{Hom}\left(Q[-1], \widetilde{I^{\bullet}}\right)=0
$$

for all 0-dimensional sheaves $Q$.

Hence $I^{\bullet}$ is a stable pair if and only $\widetilde{I^{\bullet}}$ is a $\pi$-stable pair.

3.6. Non-reduced case. We show by example that if the class $H$ is nonreduced on $S$, then there exist a stable pair $I^{\bullet}$ such that $h^{0}\left(\widetilde{I^{\bullet}}\right)$ is not an ideal sheaf. In particular the proof of Theorem 1 breaks down and new methods are required, see [28].

Example. Let $S$ be an elliptic K3 surface with a section $B \subset S$, and let $E$ be an elliptic curve. The Calabi-Yau 3-fold $X=S \times E$ admits a trivial elliptic fibration over $S$

$$
\pi: X \rightarrow S
$$

by projection. Let $F \subset S$ be a fixed fiber on the elliptic K3 surface $S$. Assume $x_{1}, x_{2}$ are two distinct points on $E$, and let $F_{i}=F \times\left\{x_{i}\right\}, i=1,2$. We consider the curve

$$
C=\iota(B) \cup F_{1} \cup F_{2} \subset X
$$

in the curve class $\iota_{*} H$ where $H=B+2 F$. The class $H$ is a primitive, but non-reduced.

Let $\mathcal{I}_{C}$ be the ideal sheaf of $C$ considered as the stable pair $\left[\mathcal{O}_{X} \rightarrow \mathcal{O}_{C}\right]$. By Lemmas 11 and 9, the complex $\widetilde{\mathcal{I}_{C}}$ is a sheaf with

$$
c_{1}\left(\widetilde{\mathcal{I}_{C}}\right)=0 \in H^{2}(X, \mathbb{Q}) .
$$

We show that $\widetilde{\mathcal{I}_{C}}$ is not an ideal sheaf.

Let $D=B+F$ and let $W=\pi^{-1}(D)$. Since $C$ lies on the surface $W$ we have the exact sequence

$$
0 \rightarrow \mathcal{O}_{X}\left(-\pi^{*} D\right) \rightarrow \mathcal{I}_{C} \rightarrow \mathcal{I}_{C / W} \rightarrow 0 .
$$

Since $\Phi_{H}\left(\mathcal{O}_{X}\left(-\pi^{*} D\right)\right)=\mathcal{O}_{X}\left(-\pi^{*} F\right)$ we obtain

$$
0 \rightarrow h^{0}\left(\widetilde{\mathcal{I}_{C / W}}\right) \rightarrow \widetilde{\mathcal{I}_{C}} \stackrel{g}{\rightarrow} \mathcal{O}_{X}\left(-\pi^{*} F\right) \rightarrow h^{1}\left(\widetilde{\mathcal{I}_{C / W}}\right) \rightarrow 0 .
$$

By definition, the sheaf $h^{0}\left(\widetilde{I_{C / W}}\right)$ is supported on the surface $W$ and hence is torsion in $X$. If $\widetilde{\mathcal{I}_{C}}$ is an ideal sheaf, then $h^{0}\left(\widetilde{I_{C / W}}\right)=0$ and thus $g$ is injective. This is a contradiction to (17) since ideal sheaves are stable.

\section{Applications}

4.1. Overview. Here we prove Theorems 2 , 3 and 6, and Proposition 1 , 
4.2. Proof of Theorem $\left[3\right.$, Let $\mathrm{N}_{n, \beta} \in \mathbb{Q}$ be the generalized DonaldsonThomas invariant counting semistable sheaves of class $(0,0, \beta, n)$. In [5, 38] the following structure result was proven:

$$
\sum_{n, \beta} \mathrm{P}_{n, \beta} q^{n} t^{\beta}=\exp \left(\sum_{n>0, \beta>0}(-1)^{n-1} n \mathrm{~N}_{n, \beta} q^{n} t^{\beta}\right) \cdot \sum_{n, \beta} \mathrm{L}_{n, \beta} q^{n} t^{\beta}
$$

where for every $\beta$ the invariants $\mathrm{L}_{n, \beta} \in \mathbb{Z}$ satisfy

- $\mathrm{L}_{n, \beta}=\mathrm{L}_{-n, \beta}$ for all $n$,

- $\mathrm{L}_{n, \beta}=0$ for all $n \gg 0$.

For every $H$ we define the series

$$
f_{H}=\sum_{d \geq 0} \sum_{n>0}(-1)^{n-1} n \mathrm{~N}_{n, H+d F} q^{n} t^{d} \quad \text { and } \quad L_{H}=\sum_{d \geq 0} \sum_{n \in \mathbb{Z}} L_{n, H+d F} q^{n} t^{d} .
$$

Let $H \in \operatorname{Pic}(S)$ be a reduced class. Picking out all $t^{H}$-terms in (18) yields

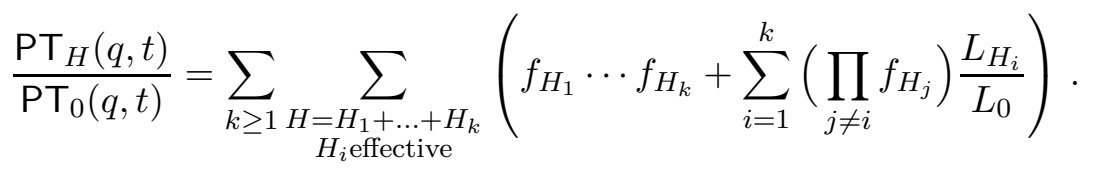

Since every summand $H_{i}$ in the above sum is non-zero and reduced we have by [35, 2.9]

$$
f_{H_{i}}=\left(q^{1 / 2}+q^{-1 / 2}\right)^{-2} \sum_{d \geq 0} \mathrm{~N}_{1, H_{i}+d F} t^{d} .
$$

Moreover by [36] we have $L_{0}=\prod_{m \geq 1}\left(1-t^{m}\right)^{-e(S)}$,

Plugging both into (19) we find that for every $d$ the $t^{d}$ coefficient of $\mathrm{PT}_{H} / \mathrm{PT}_{0}$ can be written in the following form:

$$
\left[\frac{\mathrm{PT}_{H}(q, t)}{\mathrm{PT}_{0}(q, t)}\right]_{t^{d}}=\sum_{k=-n}^{r} a_{d, k}\left(q^{1 / 2}+q^{-1 / 2}\right)^{2 k}
$$

for some $r \gg 0$ (dependent on $d$ ) and where $n$ is the largest integer for which there exist a decomposition $H=\sum_{i=1}^{n} H_{i}$ into effective classes.

On the Jacobi form side we have for every $i$

$$
\phi_{-2,1}^{i-1} \phi_{0,1}^{h-i}=12^{h-i}\left(q^{1 / 2}+q^{-1 / 2}\right)^{2 i-2}+\sum_{j=i}^{N} a_{j}\left(q^{1 / 2}+q^{-1 / 2}\right)^{2 j}+O(t)
$$

for some $a_{j} \in \mathbb{Q}$ and some $N>0$. Also every $t^{d}$-coefficient is of the form

$$
\left[\phi_{-2,1}^{i-1} \phi_{0,1}^{h-i}\right]_{t^{d}}=\sum_{k=i-1}^{r^{\prime}} a_{d, k}^{\prime}\left(q^{1 / 2}+q^{-1 / 2}\right)^{2 k} .
$$

Therefore by an induction argument there exist power series

$$
f_{-(n-1)}(t), \ldots, f_{h}(t) \in \mathbb{Q}[[t]]
$$


such that every $t^{d}$-coefficient of the series

$$
\mathcal{R}(q, t)=\frac{\mathrm{PT}_{H}}{\mathrm{PT}_{0}}-\sum_{i=-(n-1)}^{h} f_{i}(t) \varphi_{-2,1}^{i-1} \varphi_{0,1}^{h-i}
$$

takes the form

$$
\mathcal{R}_{d}(q, t)=[\mathcal{R}(q, t)]_{t^{d}}=\sum_{k=h}^{r} b_{d, k}\left(q^{1 / 2}+q^{-1 / 2}\right)^{2 k} .
$$

Equivalently, every coefficient of $\mathcal{R}_{d}(q, t)$ vanishes to order $u^{2 h}$ when expanded in the variables $q=-e^{i u}$. We prove $\mathcal{R}(q, t)=0$.

By (21) and since $h \geq 0$ by assumption we have the equality of power series

$$
\mathcal{R}\left(q^{-1}, t\right)=\mathcal{R}(q, t)
$$

Using the definition of $\phi_{-2,1}$ as a product one verifies

$$
\frac{1}{\phi_{-2,1}\left(q^{-1} t, t\right)}=q^{-2} t^{1} \frac{1}{\phi_{-2,1}(q, t)}
$$

as power series. By the Jacobi form property of $\phi_{0,1}$ [13] we also have

$$
\phi_{0,1}\left(q^{-1} t, t\right)=q^{2} t^{-1} \phi_{0,1}(q, t) .
$$

Hence we obtain for every $i \leq h$ the equality of power series

$$
\left(\phi_{-2,1}^{i-1} \phi_{0,1}^{h-i}\right)\left(q^{-1} t, t\right)=q^{2(h-1)} t^{-(h-1)}\left(\phi_{-2,1}^{i-1} \phi_{0,1}^{h-i}\right)(q, t) .
$$

Applying Theorem 1 we thus find the equality of power series:

$$
\mathcal{R}\left(q^{-1} t, t\right)=q^{2(h-1)} t^{-(h-1)} \mathcal{R}(q, t) .
$$

Combining (22) and (23) we conclude

$$
\mathcal{R}\left(q t^{\lambda}, t\right)=t^{-(h-1) \lambda^{2}} q^{-2(h-1) \lambda} \mathcal{R}(q, t)
$$

for all $\lambda \in \mathbb{Z}$ as power series. Let $c_{n, d}$ be the coefficient of $q^{n} t^{d}$ in $\mathcal{R}(q, t)$. Then (24) is equivalent to

$$
c_{n, d}=c_{n+2(h-1) \lambda, d+\lambda n+(h-1) \lambda^{2}}
$$

for all $n, d, \lambda \in \mathbb{Z}$.

Assume $\mathcal{R}(q, t)$ is non-zero and let $d$ be the smallest integer such that $\mathcal{R}_{d}(q, t)$ is non-zero. Since the sum in (21) starts at $k=h$ we have

$$
c_{n, d} \neq 0
$$

for some $n \geq h \geq 0$. But then by (25) with $\lambda=-1$ we obtain

$$
c_{n, d}=c_{n-2 h+2, d-n+(h-1)} \neq 0 .
$$

Since $d-n+(h-1)<d$ this contradicts the choice of $d$. 
4.3. Proof of Theorem [2, By Theorem 3 we have

$$
\frac{\mathrm{PT}_{H}(q, t)}{\mathrm{PT}_{0}(q, t)}=f_{0}(t) \frac{1}{\phi_{-2,1}(q, t)}
$$

for some power series $f_{0}(t)$. Hence we need to prove $f_{0}(t)=\mathrm{F}_{H}(t)$.

By (19) and (20), and since the genus 0 Gopakumar-Vafa invariant is $\mathrm{n}_{\beta}=\mathrm{N}_{1, \beta}$ we have

$$
\frac{\mathrm{PT}_{H}(q, t)}{\mathrm{PT}_{0}(q, t)}=\mathrm{F}_{H}(t) \frac{q}{(1+q)^{2}}+\frac{L_{H}}{L_{0}} .
$$

Similarly,

$$
\frac{1}{\phi_{-2,1}(q, t)}=\frac{q}{(1+q)^{2}}+\phi^{\prime}(q, t)
$$

where $\phi^{\prime}(q, t)$ is regular at $q=-1$. Comparing both sides we obtain

$$
f_{0}(t)=\mathrm{F}_{H}(t) \text {. }
$$

4.4. Proof of Proposition 1. For every $i$ we have under the variable change $q=-e^{i u}$

$$
\phi_{-2,1}^{i-1} \phi_{0,1}^{h-i}=12^{h-i} u^{2 i-2}+O\left(u^{2 i}\right) .
$$

Hence the $u^{2 i}$-coefficients of $\mathrm{PT}_{H} / \mathrm{PT}_{0}$ for $i<h$ uniquely determine the functions $f_{k}(t)$ in Theorem 3 ,

If $H$ is irreducible the coefficient of $u^{H}$ in

$$
\log \left(\sum_{L, n, d} \mathrm{P}_{n, L+d F} q^{n} t^{d} u^{L}\right)
$$

is exactly $\mathrm{PT}_{H} / \mathrm{PT}_{0}$. Therefore Proposition 1 follows from the GW/PT correspondence:

$$
\frac{\mathrm{PT}_{H}(q, t)}{\mathrm{PT}_{0}(q, t)}=\sum_{g=0}^{\infty} \mathrm{GW}_{H}^{g}(t) u^{2 g-2} .
$$

4.5. Proof of Theorem 6. By the same argument as for Theorem 3, but using the Euler characteristics case [34] of the structure result (18).

\section{EXAmPles}

5.1. Overview. We give examples of our results for the Schoen Calabi-Yau (Section 5.2), the STU-model (Section [5.3), the local case (Section 5.4), and the product case $S \times E$ (Section [5.5). For K3 $\times E$ we give the proof of Theorems 4 and 5, For $\mathrm{A} \times E$ we reprove several of the results of [10] and connect a conjecture of [10] to the modularity of the series $f_{k}(t)$. We end in Section 5.8 with an example that the Noether-Lefschetz terms $\widetilde{f}_{k}(t)$ for unweighted Euler characteristics may not be modular. 
5.2. The Schoen Calabi-Yau. A rational elliptic surface $R$ is the blowup of $\mathbb{P}^{2}$ along the 9 intersection points of two distinct integral cubics. The associated pencil of cubics defines an elliptic fibration

$$
R \rightarrow \mathbb{P}^{1} .
$$

Let $R_{1}, R_{2}$ be two generic rational elliptic surfaces. The Schoen Calabi-Yau is the fiber product

$$
X=R_{1} \times_{\mathbb{P}^{1}} R_{2} .
$$

We consider $X$ to be elliptically fibered via the projection

$$
\pi: X \rightarrow R_{1}
$$

to the first factor.

Let $B$ be the class of a section of $R_{1} \rightarrow \mathbb{P}^{1}$. By a calculation of Bryan and Leung [9, Thm 6.2] the genus 0 Gromov-Witten invariants of $X$ in classes $B+d F$ are

$$
\mathrm{GW}_{B}^{0}(t)=\sum_{d \geq 0} \mathrm{GW}_{0, B+d F}^{R_{2}} t^{d}=\prod_{m=1}^{\infty}\left(1-t^{m}\right)^{-12} .
$$

Since $e(X)=0$ and $e\left(R_{1}\right)=12$ we also have

$$
\mathrm{PT}_{0}(q, t)=\prod_{m \geq 1}\left(1-t^{m}\right)^{-12} .
$$

Using Theorem 2 we conclude

$$
\mathrm{PT}_{B}(q, t)=\frac{1}{\left(q+2+q^{-1}\right)} \prod_{m \geq 1} \frac{1}{\left(1+q t^{m}\right)^{2}\left(1-t^{m}\right)^{20}\left(1+q^{-1} t^{m}\right)^{2}} .
$$

Let $E$ be the class of an elliptic fiber of $R_{1} \rightarrow \mathbb{P}^{1}$. We have

$$
\mathrm{GW}_{E}^{0}(t)=\mathrm{GW}_{0, E}=0, \quad \mathrm{GW}_{E}^{1}(t)=\mathrm{GW}_{1, E}=12 .
$$

Hence in agreement with a stable pair calculation by J. Bryan we obtain:

$$
\mathrm{PT}_{E}(q, t)=12 \prod_{m \geq 1}\left(1-t^{m}\right)^{-12} .
$$

5.3. The STU model. The STU model is a particular non-singular CalabiYau threefold $X$ which admits an elliptic fibration

$$
\pi: X \rightarrow \mathbb{P}^{1} \times \mathbb{P}^{1}
$$

with a section. An explicit description of $X$ as a hypersurface in a toric variety can be found in [20]. The composition of $\pi$ with the projection of $\mathbb{P}^{1} \times \mathbb{P}^{1}$ to the $i$-th factor

$$
\pi_{i}: X \rightarrow \mathbb{P}^{1} \times \mathbb{P}^{1} \rightarrow \mathbb{P}^{1}
$$

is a family of K3 surfaces with 528 singular fibers. We identify

$$
\operatorname{Pic}\left(\mathbb{P}^{1} \times \mathbb{P}^{1}\right)=\mathbb{Z} \oplus \mathbb{Z}
$$


Recall the genus 0 potential $\mathrm{F}_{H}=\mathrm{F}_{H}(t)$. By [19, 20] we have

$$
\mathrm{F}_{(1,0)}(t)=\mathrm{F}_{(0,1)}(t)=\frac{-2 E_{4}(t) E_{6}(t)}{\prod_{m \geq 1}\left(1-t^{m}\right)^{24}} .
$$

The term $-2 E_{4}(t) E_{6}(t)$ is the Noether-Lefschetz contribution of the fibration (26) and counts the jumping of the Picard rank of the fibers of $\pi_{i}$. The term

$$
\prod_{m \geq 1} \frac{1}{\left(1-t^{m}\right)^{24}}
$$

is the generating series of (primitive) genus 0 invariants of $\mathrm{K} 3$ surfaces, and counts rational curves in the fibers of $\pi_{i}$. The series $\mathrm{F}_{(1,0)}(t)$ splits naturally as a product of both contributions. Applying Theorem 2 we obtain

$$
\frac{\mathrm{PT}_{(1,0)}(q, t)}{\mathrm{PT}_{0}(q, t)}=\frac{-2 E_{4}(t) E_{6}(t)}{\Delta(t) \phi_{-2,1}(q, t)},
$$

in perfect agreement with the higher genus GW/NL correspondence [22] and the Katz-Klemm-Vafa formula [18, 23].

The formula for the class $H=(1,1)$ is new and more interesting, since curves in the base may degenerate to the union of two lines. By Theorem 3 we have

$$
\frac{\mathrm{PT}_{(1,1)}(q, t)}{\mathrm{PT}_{0}(q, t)}=f_{-1}(t) \cdot \frac{\wp(q, t)}{\phi_{-2,1}(q, t)}+f_{0}(t) \cdot \frac{1}{\phi_{-2,1}(q, t)} .
$$

Studying the polar terms in (19) yields

$$
f_{-1}(t)=\mathrm{F}_{(1,0)} \mathrm{F}_{(0,1)}=4 E_{4}(t)^{2} E_{6}(t)^{2} \prod_{m \geq 1}\left(1-t^{m}\right)^{-48}
$$

and

$$
f_{0}(t)=\mathrm{F}_{(1,1)}+\frac{1}{12} E_{2}(t) \mathrm{F}_{(1,0)} \mathrm{F}_{(0,1)} .
$$

By the calculations of [19, 6.10.5] we have6,

$$
\mathrm{F}_{(1,1)}(t)=-E_{4} E_{6}\left(\frac{67}{36} E_{4}^{3}+\frac{65}{36} E_{6}^{2}+\frac{1}{3} E_{2} E_{4} E_{6}\right) \prod_{m \geq 1}\left(1-t^{m}\right)^{-48} .
$$

Hence we find

$$
f_{0}(t)=-E_{4} E_{6}\left(\frac{67}{36} E_{4}^{3}+\frac{65}{36} E_{6}^{2}\right) \prod_{m \geq 1}\left(1-t^{m}\right)^{-48} .
$$

\footnotetext{
${ }^{5}$ We assume the GW/PT correspondence for $X$ here. We expect the proof to be similar to 31 .

6 Since $X$ is a hypersurface in a toric variety, mirror symmetry for $X$ in genus 0 is proven. Hence there is no difficulty in making the genus 0 calculations of [19] rigorous.
} 
In particular, both $f_{0}(t)$ and $f_{-1}(t)$ are modular forms? We conclude that $\mathrm{PT}_{(1,1)} / \mathrm{PT}_{0}$ is a meromorphic Jacobi form.

The elliptic surface over a non-singular line $L \subset \mathbb{P}^{1} \times \mathbb{P}^{1}$ of class $(1,1)$ has 48 nodal fibers. Hence by [9] we may interpret the term

$$
\prod_{m \geq 1} \frac{1}{\left(1-t^{m}\right)^{48}}
$$

as counting rational curves in the surfaces $\pi^{-1}(L)$. The factor

$$
4 E_{4}(t)^{2} E_{6}(t)^{2}
$$

in $f_{-1}(t)$ is the product of the Noether-Lefschetz series of the family (26), and arises here naturally from the Noether-Lefschetz data of the 2-dimensional family of surfaces $\pi^{-1}(L)$ over broken lines $L=L_{1} \cup L_{2}$. We expect the factor

$$
-E_{4} E_{6}\left(\frac{67}{36} E_{4}^{3}+\frac{65}{36} E_{6}^{2}\right)=-\frac{11}{3}+1448 q-362376 q^{2}+85977632 q^{3}+\ldots
$$

to arise from the Noether-Lefschetz theory of the full family of elliptic surfaces over curves in the base.

5.4. The local case. Let $X$ be a non-singular projective threefold with $\omega_{X} \simeq \mathcal{O}_{X}$. In particular, we allow $H^{1}\left(X, \mathcal{O}_{X}\right)$ to be non-zero. Assume that $X$ admits an elliptic fibration

$$
\pi: X \rightarrow S
$$

with integral fibers over a non-singular surface $S$, and let $\iota: S \rightarrow X$ be a section. We also fix a Cohen-Macaulay curve

$$
C \subset X
$$

which satisfies the following conditions:

- The curve $C$ has no components supported on fibers of $\pi$.

- The curve $C$ is reduced.

- The restriction

$$
\left.\pi\right|_{C}: C \rightarrow D:=\pi(C)
$$

is an isomorphism away from a zero-dimensional subset $T \subset D$.

In particular, the image $D=\pi(C)$ is a reduced divisor in $S$.

Consider the closed subset

$$
P_{n}(X, C, d) \subset P_{n}(X,[C]+d F)
$$

\footnotetext{
${ }^{7}$ We ignore here the non-modularity arising from the missing $t^{-2}$ factor in the Euler product.
} 
of stable pairs $\mathcal{O}_{X} \rightarrow \mathcal{F}$ such that the support of $\mathcal{F}$ contains $C$. We define $C$-local pairs invariants by

$$
\mathrm{P}_{n, C, d}=\int_{P_{n}(X, C, d)} \nu \mathrm{d} e .
$$

where $\nu$ is the restriction of the Behrend function of $P_{n}(X,[C]+d F)$. Let

$$
\mathrm{PT}_{C}(q, t)=\sum_{d=0}^{\infty} \sum_{n \in \mathbb{Z}} \mathrm{P}_{n, C, d} q^{n} t^{d}
$$

be the generating series of $C$-local pairs invariants.

Recall also the generating series $\mathrm{PT}_{0}(q, t)$ from (1).

Theorem 8. Let $h$ be the arithmetic genus of the divisor $D \subset S$. Then in the situation above,

$$
\frac{\mathrm{PT}_{C}\left(q^{-1} t, t\right)}{\mathrm{PT}_{0}\left(q^{-1} t, t\right)}=q^{2(h-1)} t^{-(h-1)} \frac{\mathrm{PT}_{C}(q, t)}{\mathrm{PT}_{0}(q, t)} .
$$

Assume $h \geq 0$, and let $n$ be the number of rational components of $C$. Then there exist power series $f_{i}(t) \in \mathbb{Q}[[t]]$ such that

$$
\frac{\mathrm{PT}_{C}(q, t)}{\mathrm{PT}_{0}(q, t)}=\sum_{i=-(n-1)}^{h} f_{i}(t) \cdot \phi_{-2,1}(q, t)^{i-1} \phi_{0,1}(q, t)^{h-i} .
$$

Proof. We apply the same argument as in the proof of Theorem 1. The wall-crossing argument follows exactly the discussion of Section 2 but using the moduli space of $\left(\pi\right.$-)stable pairs $I^{\bullet}$ such that the curve defined by $h^{0}\left(I^{\bullet}\right)$ contains $C$. Also the argument using the derived equivalence $\Phi_{H}$ is parallel. We only need to check that the support of a stable pair $I^{\bullet}$ contains $C$ if and only if so does the curve defined by $h^{0}\left(\widetilde{I^{\bullet}}\right)$. This reduces to showing that $\widetilde{\mathcal{I}_{C}}=\mathcal{I}_{C}$. But this follows from (16), the proof of Lemma 12 and since $C$ is reduced. Hence we conclude the first claim.

The second claim follows exactly parallel to the proof of Theorem 3, with (18) replaced by the $C$-local case [25, Thm 4] and using [35, Lem 2.12].

5.5. The product case. Let $S$ be a non-singular projective surface with $\omega_{S} \simeq \mathcal{O}_{S}$. Hence $S$ is a K3 surface or an abelian surface. Let $E$ be an elliptic curve and consider the Calabi-Yau threefold

$$
X=S \times E
$$

elliptically fibered over $S$ by the projection to the first factor. Let $0_{E} \in E$ denote the zero, and fix the section

$$
\iota: S=S \times 0_{E} \hookrightarrow X .
$$

Let $H \in \operatorname{Pic}(S)$ be a divisor in the base, and let

$$
P_{n}(X,(H, d)) \subset P_{n}(X, H+d F)
$$


be the moduli space of stable pairs $(\mathcal{F}, s)$ of class $H+d F$ such that the pushforward $\pi_{*}[C]$ of the cycle of the support $C$ of $\mathcal{F}$ lies in $|H|$.

The group $\left(E, 0_{E}\right)$ acts on the moduli space by translation with finite stabilizers. We define reduced stable pair invariants of $X$ by the Behrend function weighted Euler characteristic

$$
\mathrm{P}_{n,(H, d)}^{\mathrm{red}}=\int_{P_{n}(X,(H, d)) / E} \nu \mathrm{d} e,
$$

where the Euler characteristic is taken in the orbifold sense. For $S$ an abelian surface the definition first appeared in [15], for $S$ a K3 surface it can be found in [7. Define the generating series of reduced invariants

$$
\mathrm{PT}_{H}^{\mathrm{red}}(q, t)=\sum_{d \geq 0} \sum_{n \in \mathbb{Z}} \mathrm{P}_{n,(H, d)}^{\mathrm{red}} q^{n} t^{d} .
$$

Theorem 9. Let $H \in \operatorname{Pic}(S)$ be irreducible of arithmetic genus $h$. There exist power series $f_{i}(t) \in \mathbb{Q}[[t]]$ such that

$$
\frac{\mathrm{PT}_{H}^{\mathrm{red}}(q, t)}{\mathrm{PT}_{0}(q, t)}=\sum_{i=0}^{h} f_{i}(t) \phi_{-2,1}(q, t)^{i-1} \phi_{0,1}(q, t)^{h-i} .
$$

Proof. This follows directly from Theorem 8 by integration over the quotient of the Chow variety of curves by the translation action, compare [25, 4.11].

5.6. The case $\mathrm{K} 3 \times E$. Let $S$ be a projective $\mathrm{K} 3$ surface and let

$$
H \in \operatorname{Pic}(S)
$$

be a primitive class of arithmetic genus $h$. By deformation invariance [25], the series $\mathrm{PT}_{H}^{\mathrm{red}}$ only depends on $h$. Hence we can assume $H$ is irreducible. We write

$$
\mathrm{PT}_{h}^{\mathrm{K} 3 \times E}(q, t)=\mathrm{PT}_{H}^{\mathrm{red}}(q, t) .
$$

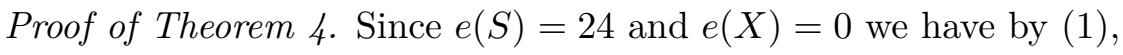

$$
\mathrm{PT}_{0}(q, t)=\frac{1}{\Delta(t)} \text {. }
$$

Hence by Theorem 9 it suffices to show that $f_{i}(t)$ are quasi-modular forms of weight $2 i$. By [25, Thm.1] the invariant $\mathrm{P}_{n, H+d F}^{\text {red }}$ is equal to the stable pair invariant of $S \times E$ defined via the reduced virtual class and insertions. Hence by [27, Prop. 5] we have the GW/PT correspondence

$$
\frac{\mathrm{PT}_{h}^{\mathrm{K} 3 \times E}(q, t)}{\mathrm{PT}_{0}(q, t)}=\sum_{d \geq 0} \sum_{g \geq 0} \mathrm{GW}_{g, H+d F} u^{2 g-2} t^{d}
$$


under the variable change $q=-e^{i u}$; here $\mathrm{GW}_{g, H+d F}$ is the reduced (connected) genus $g$ Gromov-Witten invariant of $S \times E$ in class $H+d F$ defined via the insertion $\tau_{0}\left(H^{\vee} \otimes \mathrm{pt}\right)$, see [27]. Define

$$
\mathrm{GW}_{H}^{g}(t)=\sum_{d \geq 0} \mathrm{GW}_{g, H+d F} t^{d} .
$$

By (29) the series $f_{i}(t)$ are determined by the identity

$$
\sum_{i=0}^{h} f_{i}(t) \phi_{-2,1}(q, t)^{i-1} \phi_{0,1}(q, t)^{h-i} \equiv \sum_{g=0}^{h} \mathrm{GW}_{H}^{g}(t) u^{2 g-2} \bmod u^{2 h}
$$

under the variable change $q=-e^{i u}$. Hence it is enough to show that

$$
\mathrm{GW}_{H}^{g}(t) \in \mathbb{Q}[[t]]
$$

are quasi-modular forms of weight $2 g$.

By a degeneration argument $[23,5.3]$ the series $\mathrm{GW}_{H}^{g}(t)$ can be expressed in terms of the Gromov-Witten invariants of $R \times E$, where $R$ is an rational elliptic surface. Since $R$ is deformation equivalent to a toric surface, the Gromov-Witten classes of $R$ are tautological on $\bar{M}_{g, 1}$ [14]. Hence by Behrend's product formula [4] the generating series $\mathrm{GW}_{H}^{g}$ can be expressed in terms of the following generating series of elliptic Gromov-Witten invariants:

$$
\sum_{d \geq 0}\left(\int_{\left[\bar{M}_{g, 1}(E, d)\right]^{\mathrm{vir}}} \mathrm{ev}^{*}(\mathrm{pt}) \cup h^{*} \alpha\right) q^{d},
$$

where ev $: \bar{M}_{g, 1}(E, d) \rightarrow E$ is the evaluation map, $h: \bar{M}_{g, 1}(E, d) \rightarrow \bar{M}_{g, 1}$ is the forgetful map, and $\alpha$ is a tautological class on $\bar{M}_{g, 1}$.

The induction process in the proof of [23, Prop 29] for K3 surfaces can be identically applied to the elliptic curve $E$, which allows us to write the tautological integrals (30) in terms of descendent integrals. By [29, Sec.5] we find (30) is a quasi-modular form of weight $2 g$, see also [23, Prop 28].

Proof of Theorem 5. Let $H_{0} \in \operatorname{Pic}(S)$ be irreducible of genus 0 . Then

$$
\mathrm{GW}_{H_{0}}^{0}(t)=\mathrm{GW}_{0, H_{0}}=1
$$

so by Theorem 2 and $\mathrm{PT}_{0}=\Delta^{-1}$ we obtain

$$
\mathrm{PT}_{H_{0}}(q, t)=1 \cdot \frac{1}{\Delta(t)} \cdot \frac{1}{\phi_{-2,1}(q, t)} .
$$

Let $H_{1} \in \operatorname{Pic}(S)$ be an irreducible class of genus 1 . Then

$$
\begin{aligned}
\mathrm{GW}_{H_{1}}^{0}(t) & =\mathrm{GW}_{0, H_{1}}=24 \\
\mathrm{GW}_{1, H_{1}+d F} & =24 \sigma(d) \cdot\left\langle\lambda_{1}\right\rangle_{1, H_{1}}^{S}=\sigma(d) \cdot\left(H_{1}^{2}\right) \cdot\langle 1\rangle_{0, H_{1}}^{S}=0
\end{aligned}
$$


where we used the Gromov-Witten bracket notation, the product formula, and $H_{1} \cdot H_{1}=0$ in the second line. Hence by Theorem 9 and (4) we find

$$
\mathrm{PT}_{H_{1}}(q, t)=24 \cdot \frac{1}{\Delta(t)} \cdot \wp(q, t) .
$$

5.7. Abelian threefolds. Let $A$ be an abelian surface and let

$$
H \in \operatorname{Pic}(A)
$$

be an irreducible class of arithmetic genus $h \geq 2$. By the same argument as in [25] the series $\mathrm{PT}_{H}^{\mathrm{red}}$ is invariant under deformations which preserve the product structure $A \times E$ and keep $H$ of Hodge type $(1,1)$ on $A$. Hence the series only depends on the arithmetic genus $h$ and we write

$$
\mathrm{PT}_{h}^{\mathrm{A} \times E}(q, t)=\mathrm{PT}_{H}^{\mathrm{red}}(q, t) .
$$

In [10] the Euler characteristic version of the series $\mathrm{PT}_{h}^{\mathrm{A} \times E}$ was computed in cases $h \in\{2,3\}$ via cut-and-paste methods, and a full formula was conjectured for any $h$. Using calculations on the Gromov-Witten side the case $h=2$ was proven in [26] also for the weighted case. Here we reprove the result for $h=2$, and for $h=3$ when assuming the GW/PT correspondence.

Proposition 5. $\mathrm{PT}_{2}^{\mathrm{A} \times E}(q, t)=\phi_{-2,1}(q, t)$

Proof of Proposition 5. By Theorem 9 we have

$$
\mathrm{PT}_{2}^{\mathrm{A} \times E}(q, t)=f_{0}(t) \frac{\phi_{0,1}^{2}}{\phi_{-2,1}}+f_{1}(t) \phi_{0,1}+f_{2}(t) \phi_{-2,1} .
$$

By a degeneration argument 8 the GW/PT correspondence holds for $h=2$. Hence we may compute $f_{i}(t)$ by Gromov-Witten theory. Let $H_{2} \in \operatorname{Pic}(A)$ be irreducible of genus 2 , and let

$$
\mathrm{GW}_{H_{2}}^{g}(t)=\sum_{d \geq 0} \mathrm{GW}_{g, H_{2}+d F}^{A \times E} t^{d}
$$

denote the generating series of reduced genus $g$ Gromov-Witten invariants of $A \times E$ following [10, 7.2]. Since a generic abelian surface does not admit nontrivial maps from rational or elliptic curves we have $\mathrm{GW}_{H_{2}}^{0}(t)=$ $\mathrm{GW}_{H_{2}}^{1}(t)=0$. The genus 2 invariants are

$$
\mathrm{GW}_{H_{2}}^{2}(t)=1
$$

since there is exactly one genus 2 curve in the linear system $\left|H_{2}\right|$ on $A$. Applying (51) we conclude the result.

\footnotetext{
8 The reduced virtual class splits naturally under degenerating the elliptic factor to a nodal rational curve and resolving, compare [26, 2.5] and 23, Section 4].
} 
Consider the theta function of the D4 lattice

$$
\vartheta_{\mathrm{D} 4}(t)=1+24 \sum_{d \geq 1} \sum_{\substack{k \mid d \\ k \text { odd }}} k t^{d} .
$$

Proposition 6. Let $H \in \operatorname{Pic}(A)$ be irreducible of arithmetic genus 3 , and assume the $G W / P T$ correspondence in the sense of [10, Conj. B] holds for the classes $H+d F$. Then

$$
\mathrm{PT}_{3}^{\mathrm{A} \times E}(q, t)=12 \cdot \wp(q, t) \phi_{-2,1}(q, t)^{2}-\vartheta_{\mathrm{D} 4}(t) \cdot \phi_{-2,1}(q, t)^{2} .
$$

Proof of Proposition [6. By assumption we again only need to determine the Gromov-Witten invariants $\mathrm{GW}_{H}^{g}(t)$ for genus up to 3 . The invariants vanish in $g=0,1$. By counting genus 2 curves in $A$ we obtain $\mathrm{GW}_{H}^{2}(t)=12$. For genus 3, by using lattice counting and the multiple cover formula [10] we obtain

$$
\mathrm{GW}_{H}^{3}(t)=\sum_{d \geq 1}\left(8 \sigma(2 d)+64 \sigma(d / 2) \delta_{d, \text { even }}\right) t^{d}
$$

where $\sigma(d)=\sum_{k \mid d} k$. The result follows as before by Proposition 1

For general $h$ applying Theorem 9 yields the expansion

$$
\mathrm{PT}_{h}^{\mathrm{A} \times E}(q, t)=\sum_{i=2}^{h} f_{i}(t) \phi_{-2,1}(q, t)^{i-1} \phi_{0,1}(q, t)^{h-i}
$$

for power series $f_{i}(t)$. By (and in agreement with) Conjecture $\mathrm{C}$ of [10] we have the following.

Conjecture [10]. Every series $f_{i}(t), i=2, \ldots, h$ is a modular form of weight $2 i-4$ for the congruence subgroup $\Gamma_{0}(h-1)$.

5.8. Naïve Euler characteristic. Consider as above $X=A \times E$ for an abelian surface with an irreducible class $H \in \operatorname{Pic}(A)$ of arithmetic genus 3 . Let

$$
\widetilde{\mathrm{PT}}_{H}(p, t)=\sum_{d \geq 0} \sum_{n \in \mathbb{Z}} e\left(P_{n}(X,(H, d)) / E\right) q^{n} t^{d}
$$

be the generating series of Euler characteristics of (the quotient of) the stable pair moduli space defined in (28). By [10, Thm. 5] we have

$$
\widetilde{\mathrm{PT}}_{H}(p, t)=12 \wp(-p, t) \phi_{-2,1}(-p, t)^{2}+\widetilde{f}_{3}(t) \phi_{-2,1}(-p, t)^{2}
$$

where

$$
\tilde{f}_{3}(t)=1+12 \sum_{d \geq 1} \sum_{k \mid d}\left(2 k t^{d}+k t^{2 d}\right)=\frac{5}{2}-E_{2}(t)-\frac{1}{2} E_{2}\left(t^{2}\right) .
$$

In particular, $\widetilde{f}_{3}(t)$ is not modular. 


\section{REFERENCES}

[1] B. Andreas and D. Hernández Ruipérez, Fourier Mukai transforms and applications to string theory, Rev. R. Acad. Cien. Serie A. Mat. 99(1) (2005), no. 3, 29-77.

[2] C. Bartocci, U. Bruzzo, D. Hernández Ruipérez, J. Muñoz Porras, Relatively stable bundles over elliptic fibrations, Math. Nachr. 238 (2002), 23-36. Revised version available from arXiv:0109123.

[3] K. Behrend, Donaldson-Thomas type invariants via microlocal geometry, Ann. of Math. (2) 170 (2009), no. 3, 1307-1338.

[4] K. Behrend, The product formula for Gromov-Witten invariants, J. Algebraic Geom. 8 (1999), no. 3, 529-541.

[5] T. Bridgeland, Hall algebras and curve-counting invariants, J. Amer. Math. Soc. 24 (2011), no. 4, 969-998.

[6] T. Bridgeland and A. Maciocia, Fourier-Mukai transforms for K3 and elliptic fibrations, J. Algebraic Geom. 11 (2002), no. 4, 629-657.

[7] J. Bryan, The Donaldson-Thomas theory of $K 3 \times E$ via the topological vertex, arXiv:1504.02920.

[8] J. Bryan and M. Kool, Donaldson-Thomas invariants of local elliptic surfaces via the topological vertex, arXiv:1608.07369.

[9] J. Bryan and N. C. Leung, The enumerative geometry of K3 surfaces and modular forms, J. Amer. Math. Soc. 13 (2000), no. 2, 371-410.

[10] J. Bryan, G. Oberdieck, R. Pandharipande, and Q. Yin, Curve counting on abelian surfaces and threefolds, arXiv:1506.00841

[11] J. Bryan and D. Steinberg, Curve counting invariants for crepant resolutions, Trans. Amer. Math. Soc. 368 (2016), no. 3, 1583-1619.

[12] I. Burban and B. Kreussler, Fourier-Mukai transforms and semi-stable sheaves on nodal Weierstraß cubics, J. Reine Angew. Math. 584 (2005), 45-82.

[13] M. Eichler and D. Zagier, The theory of Jacobi forms, volume 55 of Progress in Mathematics, Birkhäuser Boston Inc., Boston, MA, 1985.

[14] C. Faber and R. Pandharipande, Relative maps and tautological classes, J. Eur. Math. Soc. 7 (2005), no. 1, 13-49.

[15] M. G. Gulbrandsen, Donaldson-Thomas invariants for complexes on abelian threefolds, Math. Z. 273 (2013), no. 1-2, 219-236.

[16] M. Huang, S. Katz, A. Klemm, Topological string on elliptic CY 3-folds and the ring of Jacobi forms, J. High Energy Phys. 2015, no. 10, 125.

[17] S. Katz, Genus zero Gopakumar-Vafa invariants of contractible curves, J. Differential Geom. 79 (2008), no. 2, 185-195.

[18] S. Katz, A. Klemm, and C. Vafa, M-theory, topological strings, and spinning black holes, Adv. Theor. Math. Phys. 3 (1999), 1445-1537.

[19] A. Klemm, M. Kreuzer, E. Riegler, E. Scheidegger, Topological string amplitudes, complete intersection Calabi-Yau spaces and threshold corrections, J. High Energy Phys. 2005, no. 5, 023, 116 pp.

[20] A. Klemm, D. Maulik, R. Pandharipande, E. Scheidegger, Noether-Lefschetz theory and the Yau-Zaslow conjecture, J. Amer. Math. Soc. 23 (2010), no. 4, 1013-1040.

[21] J. Lo, Polynomial Bridgeland Stable Objects and Reflexive Sheaves, Math. Res. Lett. 19 (2012), 873-885.

[22] D. Maulik and R. Pandharipande, Gromov-Witten theory and Noether-Lefschetz theory, in A celebration of algebraic geometry, Clay Mathematics Proceedings 18, 469507, AMS (2010).

[23] D. Maulik, R. Pandharipande, and R. Thomas, Curves on K3 surfaces and modular forms, J. of Topology 3 (2010), 937-996.

[24] G. Oberdieck, Gromov-Witten invariants of the Hilbert scheme of points of a K3 surface, arXiv:1406.1139. 
[25] G. Oberdieck, On reduced stable pair invariants, arXiv:1605.04631.

[26] G. Oberdieck, Gromov-Witten theory of $K 3 \times \mathbb{P}^{1}$ and Quasi-Jacobi forms, arXiv:1605.05238

[27] G. Oberdieck and R. Pandharipande, Curve counting on $K 3 \times E$, the Igusa cusp form $\chi_{10}$, and descendent integration, in K3 surfaces and their moduli, C. Faber, G. Farkas, and G. van der Geer, eds., Birkhauser Prog. in Math. 315 (2016), 245-278.

[28] G. Oberdieck and J. Shen, Curve counting on elliptic Calabi-Yau threefolds via derived categories $I I$, in preparation.

[29] A. Okounkov and R. Pandharipande, Gromov-Witten theory, Hurwitz theory, and completed cycles, Ann. of Math. (2) 163 (2006), no. 2, 517-560.

[30] R. Pandharipande and A. Pixton, Gromov-Witten/pairs descendent correspondence for toric 3-folds, Geom. Topol. 18 (2014), no. 5, 2747-2821.

[31] R. Pandharipande and A. Pixton, Gromov-Witten/Pairs correspondence for the quintic 3-fold, arXiv:1206.5490.

[32] R. Pandharipande and R. P. Thomas, Curve counting via stable pairs in the derived category, Invent. Math. 178 (2009), no. 2, 407-447.

[33] R. Pandharipande and R. P. Thomas, 13/2 ways of counting curves, in Moduli spaces, 282-333, London Math. Soc. Lecture Note Ser., 411, Cambridge Univ. Press, Cambridge, 2014.

[34] Y. Toda, Generating functions of stable pair invariants via wall-crossings in derived categories, New developments in algebraic geometry, integrable systems and mirror symmetry (RIMS, Kyoto, 2008), 389-434, Adv. Stud. Pure Math., 59, Math. Soc. Japan, Tokyo, 2010.

[35] Y. Toda Multiple cover formula of generalized DT invariants II: Jacobian localizations, arXiv:1108.4993.

[36] Y. Toda, Stability conditions and curve counting invariants on Calabi-Yau 3-folds, Kyoto J. Math. 52 (2012), no. 1, 1-50.

[37] Y. Toda, Stable pair invariants on Calabi-Yau threefolds containing $\mathbb{P}^{2}$, Geom. Topol. 20 (2016), no. 1, 555-611.

[38] Y. Toda, Hall algebras in the derived category and higher rank DT invariants, arXiv:1601.07519.

Mit, Department of Mathematics

E-mail address: georgo@mit.edu

ETH ZÜRICh, Department of Mathematics

E-mail address: junliang.shen@math.ethz.ch 